\title{
Increasing linkages among European regions. The role of sectoral composition $^{\text {is }}$
}

\author{
María Dolores Gadea-Rivas ${ }^{\mathrm{a}}$, Ana Gómez-Loscos ${ }^{\mathrm{b}}$, Danilo Leiva-Leon ${ }^{\mathrm{b}, *}$ \\ a Universidad de Zaragoza, Spain \\ ${ }^{\mathrm{b}}$ Banco de España, Spain
}

\section{A R T I C L E I N F O}

JEL classification:

C31

C32

E32

R11

\section{Keywords:}

Business cycle

Sectoral composition

Regime-switching

Model averaging

\begin{abstract}
A B S T R A C T
This paper analyses changes in economic regional interlinkages in Europe over time and investigates the factors that could explain the dynamics of these changes. Our four main findings are the following: (i) we detect a significant surge in regional synchronisation after the Great Recession; (ii) we identify the regions most interrelated with the rest of Europe, namely, Ile de France, Inner London and Lombardia; (iii) we find that sectoral composition explains regional synchronisation in Europe, mainly after the Great Recession and (iv) we document that sectoral composition has important implications for aggregate economic fluctuations, in particular, that similarities in services-related sectors across regions explain a nonlinear relationship between sectoral composition and regional business cycle synchronisation. We also propose a new method to measure time-varying synchronisation in small samples that combines regime-switching models and dynamic model averaging.
\end{abstract}

\section{Introduction}

The regional dimension has been a key concern for European institutions since the establishment of the European Community. Indeed, the regional policy of the European Union (EU) currently makes up around one third of the total EU budget and, since the Treaty of Rome in 1957, has been focused on diminishing regional disparities. ${ }^{1}$

Analysing regional economic linkages in Europe is important because it helps to provide a deeper risk assessment in policy making, given their implications in the design of European convergence policies and in the design of national fiscal policies. Furthermore, the study of spillovers plays an important role in the allocation of resources, such as infrastructure projects.

Business cycle relationships may have changed over time due to structural factors. For instance, a common pattern is that, as economies develop, they tend to specialize in the provision of services and to reduce the relevance of agriculture (see Herrendorf et al., 2014). These changes over time in economic structure may bring about changes in the degree of business cycle synchronisation.

It is worth noting that Europe has not only experienced significant policy changes to strengthen its unification process, but has also been exposed to large business cycle shocks, coming from the external side, during the Great Recession, and from the domestic side, during the recent 'Debt Crisis'. These factors may have also produced significant changes in the overall patterns of regional business cycle synchronisation.

Within this framework, the aim of this paper is twofold. On the one hand, to study the dynamics of regional business cycle linkages in Europe; on the other, to find out the mechanisms that explain these dynamics, focusing on those related to changes in sectoral composition. Our work has some advantages over the previous literature and provides a threefold contribution. First, we use data with a broader time

\footnotetext{
\& The views in this paper are those of the authors and do not represent the views of the Banco de España or the Eurosystem. We thank L. J. Alvarez and

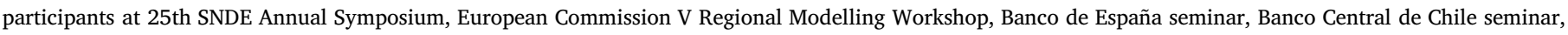

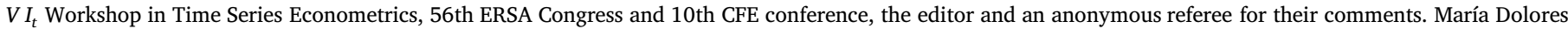

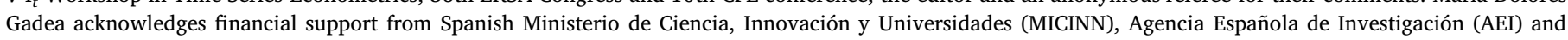

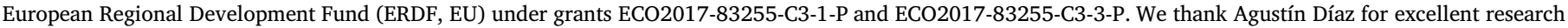
assistance.

* Corresponding author.

E-mail addresses: lgadea@unizar.es (M.D. Gadea-Rivas), agomezloscos@bde.es (A. Gómez-Loscos), danilo.leiva@bde.es (D. Leiva-Leon).

1 http://ec.europa.eu/regional_policy/en/policy/what/investment-policy/.
} 
and space coverage than previous studies, considering 213 European regions based on the NUTS2 classification (Nomenclature of territorial units for statistics, at the second level) and corresponding to 16 European countries for a period of 35 years (1980-2014) with a yearly frequency. ${ }^{2}$ This allows us to study the effects of the Great Recession and the European debt crisis on regional synchronisation and to compare their impact to other major milestones, such as the Maastricht Treaty and the introduction of the euro. Most of the previous studies look at a smaller number of European regions (NUTS1 level) and a shorter period of time. ${ }^{3}$ Finally, we employ the most comprehensive measure of real economic activity, that is, real GDP data, as the literature on national business cycle synchronisation usually does.

Second, we propose a methodology to measure time-varying synchronisation in small samples to overcome the short length of the series resulting from the absence of high frequency data. In doing so, we combine the regime-switching synchronisation approach proposed in Leiva-Leon (2016) with the dynamic model averaging framework for Markov-switching models, proposed in Guerin and Leiva-Leon (2017), to provide a flexible framework that allows us to estimate business cycle time-varying synchronisation. Monte Carlo simulations validate the reliability of the proposed framework when dealing with scarce information in the time dimension. ${ }^{4}$ Our approach not only deals with this limitation, but also provides measures of uncertainty for the estimated dynamic synchronisations.

Third, our framework is able to endogenously identify changes in regional synchronisation patterns over time and to provide assessments about the main factors associated with those changes. With the information on bilateral business cycle linkages, we can identify the most central regions in the propagation of business cycle shocks in Europe. We also analyse the role played by structural transformations in explaining business cycle synchronisation. ${ }^{5}$ Specifically, we evaluate the relationship between changes in sectoral composition and business cycle similarities over time.

In spite of the importance of the analysis of regional business cycles and their interdependence, ${ }^{6}$ the attention given to this issue has been scarce. In contrast, numerous studies have examined the business cycle and the degree of bilateral synchronisation among countries within the European Monetary Union and among European countries in general, see Camacho et al. (2006), Giannone et al. (2010) and de Haan et al. (2008) for a survey.

Most of the studies that have focused on describing overall regional cyclical patterns can be divided into two strands. The first focuses on analysing regional convergence (Ramajo et al. (2008) and Sala-i-Martin (1996)) and on identifying the determinants of long-term economic performance at the regional level (Ozyurt and Dees (2015) and RodriguezPose (2013)). The second, which is directly related to our work, focuses on the synchronisation of short-term fluctuations in regional real activity. Unlike our approach, most of the papers that analyse synchronisation among European regions use standard pairwise correlations and consider different measures of economic activity. For instance, Fatas (1997) and Barrios and De Lucio (2003) use employment data,

2 The NUTS 2013 classification lists 98 regions at NUTS1 level and 276 regions at NUTS2 level. The regions eligible for the support of the Cohesion Policy are defined at NUTS 2 level.

3 Barrios and De Lucio (2003) include 20 Spanish and Portuguese regions for 1988-1998; Fatas (1997) uses 38 regions from 4 countries for 1966-1992; Acedo-Montoya and de Haan (2011) consider 53 regions from 12 countries for 1975-2005; and Marino (2013) employs 107 regions (30 NUTS1 and 77 NUTS2 regions) from 9 countries for 1977-1995.

${ }^{4}$ An alternative rolling approach is not suitable in this case because of the small number of observations.

${ }^{5}$ For instance, Imbs (2004) and Ductor and Leiva-Leon (2016) show that similarities in sectoral composition play an important role in determining international business cycle synchronisation.

${ }^{6}$ See Gadea et al. (2012). while Acedo-Montoya and de Haan (2011) and Barrios et al. (2003) use gross value added and Clark and van Wincoop (2001) employ both measures of real economic activity to compare synchronisation patterns among European countries and US Census regions. ${ }^{7}$ Some papers compare regional cycles with country-specific cycles or with a reference cycle, such as the European one. ${ }^{8}$ Marino (2013) uses dynamic factor models to analysis regional GDP and employment fluctuations. However, dynamic factor models may not be a well suited approach to provide an assessment of regional business cycles bilateral relationships. This is because factor models rely on the decomposition of real activity into common and idiosyncratic components, although they typically assume that the idiosyncratic components are independent from each other, thus precluding the study of regional bilateral linkages.

The main findings of our analysis are the following. First, we identify a broad upward trend in synchronisation among European regions over time. In only two years, the Great Recession led to a significant increase in the level of regional synchronisation that was about twice as large as the one associated with the European Union integration process until then. After the Great Recession, the level of regional synchronisation experienced a decline, but it remained at significantly higher levels than before this event. Second, some regions, such as Ile de France, followed by Inner London and Lombardia, are more interrelated with the rest, playing a particular role in the transmission of business cycle shocks. Third, we document a gradual but persistent increase in the similarities of regional sectoral composition over time. This result has important implications for the propagation of shocks throughout the European economy because the more similar the economic structure among regions is, the more similar would be their responsiveness to shocks, potentially amplifying their effects at the aggregate level. Fourth, we find that the similarities of regional sectoral composition have become significantly more important in explaining regional synchronisation in Europe since the Great Recession. Moreover, we document a nonlinear relationship between sectoral composition and regional business cycle synchronisation, which is mainly explained by services-related sectors. Thus, this structural change has significant implications for the evolution of aggregate economic fluctuations.

The remainder of the paper is organized as follows. Section 2 presents the methodology for measuring time-varying synchronisation and assesses its reliability with simulated data. Section 3 describes the dataset and analyses regional business cycle synchronisation patterns. Section 4 investigates the relationship between the regional synchronisation and similarities in sectoral composition. Finally, Section 5 concludes.

\section{Measuring regional business cycle synchronisation}

Regime-switching models have been widely used to infer endogenous changes over time in the synchronisation of business cycle phases, at the country level (Ductor and Leiva-Leon (2016)), and at the regional (Leiva-Leon (2016)) and sectoral level (Camacho and Leiva-Leon (2017)) for the US. However, the econometric framework used in these studies requires relatively large time spans in order to provide inferences on changes of synchronisation regimes (e.g. high or low). This is an important limitation to analyse endogenous changes in the synchronisation of European regional business cycles because regional real GDP data is available yearly from 1980 to 2014, leaving only 35 observations in the time dimension of our analysis. Alternative rolling approaches are not suitable for analysing endogenous changes

\footnotetext{
${ }^{7}$ For a summary review of this literature, see Acedo-Montoya and de Haan (2011).

8 For the US, Hamilton and Owyang (2012) study similarities and differences across US states finding clusters of states sharing certain business cycle characteristics. For East Asia, Allegret and Essaadi (2011) and Dufrenot and Keddad (2014) analyse business cycle comovements.
} 
in synchronisation in the present case because of there are few observations. In this section, we propose a framework that allows us to overcome the limitations of the short sample and provide an estimation of the changes in synchronisation.

\subsection{The model}

To measure business cycle synchronisation, we follow Harding and Pagan (2006) on testing the hypothesis that cycles are either unsynchronised or perfectly synchronised during a given sample period. In this section, we propose an econometric framework to assess the degree of business cycle synchronisation over time using Dynamic Model Averaging to account for both polar cases of synchronisation. Let $Y_{a, t}$ and $Y_{b, t}$ be the real GDP of European regions " $a$ " and " $b$ ", respectively. To focus on cyclical fluctuations, we define $y_{k, t}=100 \times \Delta \log \left(Y_{k, t}\right)$ as the real GDP growth of region " $k$ ", and model their joint dynamics following Leiva-Leon (2016), that is

$\left[\begin{array}{l}y_{a, t} \\ y_{b, t}\end{array}\right]=\left[\begin{array}{l}\mu_{a}\left(S_{a, t}\right) \\ \mu_{b}\left(S_{b, t}\right)\end{array}\right]+\left[\begin{array}{l}e_{a, t} \\ e_{b, t}\end{array}\right], \quad\left[\begin{array}{l}e_{a, t} \\ e_{b, t}\end{array}\right] \sim N(0, \Sigma)$

where

$\mu_{k}\left(S_{k, t}\right)=\mu_{k, 0}+\mu_{k, 1} S_{k, t}$, for $k=a, b$,

and $S_{k, t}$ denotes a latent state variable that indicates the state of the economy of region $k$. It takes the value $S_{k, t}=0$ if region $k$ is in a low growth regime, which can be interpreted as a recessionary phase, or the value of $S_{k, t}=1$ if region $k$ is in a high growth regime, which can be interpreted as an expansionary phase. Each state variable is assumed to follow a first-order Markov process with transition probabilities given by

$p\left(S_{k, t}=j_{k} \mid S_{k, t-1}=i_{k}, S_{k, t-2}=h_{k}, \ldots\right)=p\left(S_{k, t}=j_{k} \mid S_{k, t-1}=i_{k}\right)$,

and the variance covariance matrix $\Sigma$ is assumed to be non-diagonal.

The dependency relationship between the state variables provides information about the synchronisation of the economic cycles of the two regions. Despite the complexity involved in modelling the exact dependency relationship between $S_{a, t}$ and $S_{b, t}$, we are able to model their joint dynamics, summarized in the state variable $S_{a b, t}$, under the two extreme cases. ${ }^{9}$ The first corresponds to the independent case, where the joint probability is the product of the marginal probabilities,

$p\left(S_{a b, t}^{I}=i_{a b}\right)=p\left(S_{a, t}=i_{a}\right) p\left(S_{b, t}=i_{b}\right)$.

The second corresponds to the fully dependent case, where $S_{a, t}=S_{b, t}$, = $S_{t}$, and the joint probability is modelled as

$p\left(S_{a b, t}^{D}=i_{a b}\right)=p\left(S_{t}=i\right)$,

where $S_{t}$ is a state variable that governs the whole dynamics of the system in Equation (1) and has its own transition probabilities

$p\left(S_{t}=j \mid S_{t-1}=i, S_{t-2}=h, \ldots\right)=p\left(S_{t}=j \mid S_{t-1}=i\right)$.

Our goal is to provide assessments about the degree of business cycle synchronisation between regions " $a$ " and " $b$ ", which can be interpreted as a linear combination between the two extreme cases. Moreover, we are interested in providing information about the degree of synchronisation for each period of time. Therefore, we model the joint probability of state variables as follows

$p\left(S_{a b, t}=i_{a b}\right)=p\left(S_{a b, t}^{D}=i_{a b}\right) \delta_{t}^{a b}+p\left(S_{a b, t}^{I}=i_{a b}\right)\left(1-\delta_{t}^{a b}\right)$,

where the weight $\delta_{t}$ can be interpreted as the degree of synchronisation between the two regions at time $t$. To model $\delta_{t}$, Leiva-Leon (2016) introduces another state variable that indicates either regimes

${ }^{9}$ Phillips (1991) and Bengoechea et al. (2006) model the synchronisation between two economies as a combination of two extreme situations: independent and fully dependent. where the independent case, $p\left(S_{a b, t}^{I}=i_{a b}\right)$, provides a better characterization of the model's dynamics or regimes where the dependent case, $p\left(S_{a b, t}^{D}=i_{a b}\right)$, is the most appropriate characterization. However, inferences about the time-varying synchronisation using this framework are less accurate when the number of observations contained in $y_{t}$ is very limited. This is because synchronicity regimes (independent or fully dependent) are considered to be a sequence of several time periods where the latent variables follow specific dynamics. Due to the data limitations at regional level, our available information consists of only 34 data points in the time dimension (after computing growth rates).

To overcome this drawback, in this paper, we propose a flexible way to compute $\delta_{t}^{a b}$, which is not based on the assumption of regimes of dependency. Instead, we use Dynamic Model Averaging to infer time periods where one polar case, the independent or fully dependent, provides the best characterization of the data in $y_{t}$. This procedure allows us to capture changes in European regional synchronisation with the 34 observations available at a yearly frequency.

\subsection{Dynamic model averaging}

Dynamic model averaging (DMA) was initially proposed by Raftery et al. (2010) and motivated by taking into account time variation in model uncertainty. DMA has been applied in the context of time-varying parameter (TVP) regression models (Koop and Korobilis (2012)), linear vector autoregressive (VAR) models (Koop (2014)) and large TVP-VAR models (Koop and Korobilis (2013)) to forecast inflation, real output and interest rates. Recently, Guerin and Leiva-Leon (2017) proposed an algorithm to use DMA in the context of Markov-switching (MS) models and use it to predict recessions under model uncertainty. Accordingly, we can think of the real GDP growth of regions " $a$ " and " $b$ ", $\left(y_{a, t}, y_{b, t}\right)^{\prime}$, being modelled either under the assumption of two independent business cycles driving each economy, $p\left(S_{a b, t}^{I}=i_{a b}\right)$ or under the assumption of only one common business cycle driving both economies, $p\left(S_{a b, t}^{D}=i_{a b}\right)$. In this context, the time-varying weight, $\delta_{t}^{a b}$, allows us to deal with model uncertainty and to assess which specification provides a better fit of the data for each period of time. Therefore, we can interpret our synchronisation framework in Equation (7) as a dynamic average of two MS models and follow the line of Guerin and Leiva-Leon (2017) to compute $\delta_{t}^{a b}$.

Taking the model's parameters as known and suppressing the indexes " $a$ " and " $b$ " for ease of notation, the algorithm used to obtain the elements in equation (7) consists of iteratively computing the following steps.

Step 1: predicting regime probability. Using the corresponding transition probabilities for the independent model, $p^{I}=p\left(S_{a, t} \mid\right.$ $\left.S_{a, t-1}\right) \times p\left(S_{b, t} \mid S_{b, t-1}\right)$, and for the fully dependent model, $p^{D}=p\left(S_{t} \mid\right.$ $S_{t-1}$ ), as defined in equations (3) and (6), respectively, compute the predicted regime probabilities, $p\left(S_{t}^{m} \mid, \psi_{t-1}\right)$, for $m=I, D$, given past information $\psi_{t-1}$, being $j=[0,1]$ and $i=[0,1] .{ }^{10}$

$p\left(S_{t}^{m}=j, S_{t-1}^{m}=i \mid \psi_{t-1}\right)=p^{m} p_{i j}\left(S_{t-1}^{m}=1 \mid \psi_{t-1}\right)$

$p\left(S_{t}^{m}=j \mid \psi_{t-1}\right)=\sum_{S_{t-1}^{m}} p\left(S_{t}^{m}=j, S_{t-1}^{m}=i \mid \psi_{t-1}\right)$

Then, the predictive likelihood is calculated from the predicted regime probabilities:

$$
\begin{aligned}
f_{m}\left(y_{t} \mid \psi_{t-1}\right)= & \sum_{S_{t}^{m}} \sum_{S_{t-1}^{m}} f_{m}\left(y_{t} \mid S_{t}^{m}=j, S_{t-1}^{m}=i, \psi_{t-1}\right) \\
& \times p\left(S_{t}^{m}=j, S_{t-1}^{m}=i \mid \psi_{t-1}\right) .
\end{aligned}
$$

\footnotetext{
10 The Hamilton filter is initialized with the ergodic probabilities $P\left(S_{0}\right)$.
} 
Step 2: updating model probability. Let $M_{t} \in\{I, D\}$ be a variable that indicates the model that applies at each period of time. Also, to simplify notation, let $\pi_{t \mid t-1, m}=p\left(M_{t}=m \mid \psi_{t-1}\right)$ be the predictive probability associated with the $m$-th MS model at time $t$, given the information up to $t-1$. Starting with an equal-weight initial-model probability $p\left(M_{0}\right)$, we follow the updating criterion of Raftery et al. (2010), which is based on a measure of model fit for $y_{t}$, that is, the predictive likelihood:

$\pi_{t \mid t, m}=\frac{\pi_{t \mid t-1, m} f_{m}\left(y_{t} \mid \psi_{t-1}\right)}{\sum_{r=1}^{2} \pi_{t \mid t-1, r} f_{r}\left(y_{t} \mid \psi_{t-1}\right)}$.

Step 3: updating regime probability. Use the predictive likelihood, $f_{m}\left(y_{t} \mid \psi_{t-1}\right)$, to compute the updated regime probabilities, $p\left(S_{t}^{m} \mid\right.$ $\psi_{t}$ ), for both models, as follows:

$$
\begin{aligned}
p\left(S_{t}^{m}=j, S_{t-1}^{m}=i \mid \psi_{t}\right) & =\frac{f_{m}\left(y_{t}, S_{t}^{m}=j, S_{t-1}^{m}=i \mid \psi_{t-1}\right)}{f_{m}\left(y_{t} \mid \psi_{t-1}\right)} \\
& =\frac{f_{m}\left(y_{t} \mid S_{t}^{m}=j, S_{t-1}^{m}=i, \psi_{t-1}\right) p\left(S_{t}^{m}=j, S_{t-1}^{m}=i \mid \psi_{t-1}\right)}{f_{m}\left(y_{t} \mid \psi_{t-1}\right)},
\end{aligned}
$$

$p\left(S_{t}^{m} \mid \psi_{t}\right)=\sum_{S_{t-1}^{m}} p\left(S_{t}^{m}=j, S_{t-1}^{m}=i \mid \psi_{t}\right)$

These are used in Step 1 of the next iteration.

Step 4: predicting model probability. Compute the predicted probability associated with the $m$-th model, $\pi_{t+1 \mid t, m}$, following Raftery et al. (2010) and using the forgetting factor $\alpha$, as follows:

$\pi_{t+1 \mid t, m}=\frac{\pi_{t \mid t, m}^{\alpha}}{\sum_{r=1}^{2} \pi_{t \mid t, r}^{\alpha}}$

This is used in Step 2 of the next iteration. The forgetting factor $\alpha$ is the coefficient that governs the amount of persistence in the models' weights. The higher the $\alpha$, the greater the weight attached to past predictive performance. It is commonly set to a fixed value slightly less than one. However, in our context, due to the small sample size, small variations in $\alpha$ may influence the dynamics of $\pi_{t+1 \mid t, m}$. Therefore, instead of simply imposing a given forgetting factor, we estimate it along with the other parameters of the model using Bayesian methods.

We repeat the steps above for $t=1, \ldots, T$. The output of the algorithm consists of the regime probabilities for each model, $p\left(S_{t}^{m} \mid \psi_{t}\right)$, for $m=I, D$, and the model probabilities for each time period, $\pi_{t \mid t, m}$. Therefore, we compute the expected joint regime probabilities by averaging across models:

$p\left(S_{a b, t}=i_{a b} \mid \psi_{t}\right)=p\left(S_{t}^{D} \mid \psi_{t}\right) \pi_{t \mid t, D}+p\left(S_{t}^{I} \mid \psi_{t}\right) \pi_{t \mid t, I}$,

where $\pi_{t \mid t, D}=\delta_{t \mid t}$, and $\pi_{t \mid t, I}=\left(1-\delta_{t \mid t}\right)$, following the notation in equation (7). Another advantage of the proposed approach is that it does not only provide a point estimate of the degree of synchronisation, but is also able to compute the entire distribution of the synchronisation at a given time period. From which we can obtain a measure of uncertainty associated with the estimated synchronisation.

\subsection{Estimation}

Since the likelihood function of the model in equations (1)-(7) is conditional on many possible states, the parameter estimation obtained with the maximum likelihood approach could become cumbersome. Therefore, we use a Bayesian approach to estimate this model. In particular, the approach to estimate the vector of the model's parameters, $\theta$, is based on a multivariate extended version of the multi-move Gibbssampling procedure developed by Kim and Nelson (1999). In this setting, (i) the parameters of the model, $\theta$, (ii) the Markov-switching variables, $\widetilde{S}_{a, T}=\left\{S_{a, t}\right\}_{1}^{T}, \widetilde{S}_{b, T}=\left\{S_{b, t}\right\}_{1}^{T}, \widetilde{S}_{T}=\left\{S_{t}\right\}_{1}^{T}$, and (iii) the synchronisation measure, $\widetilde{\delta}_{T}=\left\{\delta_{t}\right\}_{1}^{T}$, are treated as random variables given the data, $\tilde{y}_{T}=\left\{y_{t}\right\}_{1}^{T}$. The purpose of this Markov chain Monte Carlo simulation method is to proxy the joint and marginal distributions of these random variables by sampling from conditional distributions.

The Gibbs sampler used in the estimation procedure can be briefly described in the following steps:

Step 1: Generate the latent variables, $\widetilde{S}_{a, T}, \widetilde{S}_{b, T}, \widetilde{S}_{T}$, and the weights, $\widetilde{\delta}_{T}$, conditional on the data, $\tilde{y}_{T}$, and the vector of parameters, $\theta$.

Step 2: Generate the transition probabilities associated with each latent variable, $p_{00, a}, p_{11, a}, p_{00, b}, p_{11, b}, p_{00}, p_{11}$, conditional on $\widetilde{S}_{a, T}$, $\widetilde{S}_{b, T}, \widetilde{S}_{T}$, and $\widetilde{\delta}_{T}$.

Step 3: Generate the means associated with each state, $\mu_{a, 0}, \mu_{a, 1}$, $\mu_{b, 0}, \mu_{b, 1}$, conditional on $\sigma_{a}^{2}, \sigma_{b}^{2}, \sigma_{a b}, \widetilde{S}_{a, T}, \widetilde{S}_{b, T}, \widetilde{S}_{T}, \widetilde{\delta}_{T}$ and $\tilde{y}_{T}$.

Step 4: Generate the variance-covariance matrix, with elements $\sigma_{a}^{2}$, $\sigma_{b}^{2}, \sigma_{a b}$, conditional on $\mu_{a, 0}, \mu_{a, 1}, \mu_{b, 0}, \mu_{b, 1}, \widetilde{S}_{a, T}, \widetilde{S}_{b, T}, \widetilde{S}_{T}, \widetilde{\delta}_{T}$ and $\tilde{y}_{T}$.

Step 5: Generate the forgetting factor, $\alpha$, conditional on $\widetilde{\delta}_{T}$.

Steps 1 through 5 can be iterated $L+M$ times, where $L$ is large enough to ensure that the Gibbs sampler has converged. Thus, the marginal distributions of the state variables, the synchronisation variable and the parameters of the model can be proxied by the empirical distribution of the $M$ simulated values. We set $M=6000$ and $L=1000$. For detailed information about each step of the Gibbs sampler and the prior distributions employed, see Appendix A.

\subsection{Simulations}

In this section, we conduct Monte Carlo simulations to compare the finite sample performance of the method proposed in this paper to measure time-varying synchronisation and the method proposed in Leiva-Leon (2016) for the same purpose. Our goal is to examine the accuracy of the two methods in inferring changes in synchronisation under different scenarios, which consider (i) the gap between the statedependent parameters, (ii) the volatility of the series, and (iii) the size of the sample.

\subsubsection{Design}

The experiment consists of generating two series, $y_{a, t}$ and $y_{b, t}$, governed by two latent state variables, $S_{a, t}$ and $S_{b, t}$, respectively, in accordance with the following parsimonious system,

$\left[\begin{array}{l}y_{a, t} \\ y_{b, t}\end{array}\right]=\left[\begin{array}{l}\mu_{S_{a, t}} \\ \mu_{S_{b, t}}\end{array}\right]+\left[\begin{array}{l}e_{a, t} \\ e_{b, t}\end{array}\right]$,

where $\mu_{S_{i, t}}=\mu_{i, 0}+\mu_{i, 1} S_{i, t}$, for $i=a, b$, and the innovations $e_{t}=\left[e_{a, t}, e_{b, t}\right]^{\prime}$, are normally distributed, that is, $e_{t} \sim N(0, \Omega)$. The latent state variables are assumed to undergo changes in their synchronisation over time, which are generated as follows.

First, let $\widetilde{S}_{a, t}$ be a state vector of sequence $a$ at time $t$. If the sequence $a$ is in state 1 at time $t$, we write $\widetilde{S}_{a, t}=(1,0)^{\prime}$, and if it is in state 2 at time $t$, we write $\widetilde{S}_{a, t}=(0,1)^{\prime}$. The vector $\widetilde{S}_{a, t}$ is assumed to follow a firstorder Markov chain. For time $t$, compute $\left(q_{a}, 1-q_{a}\right)^{\prime}=P_{a} \widetilde{S}_{a, t}$, where

$P_{a}=\left(\begin{array}{cc}p_{a, 11} & 1-p_{a, 22} \\ 1-p_{a, 11} & p_{a, 22}\end{array}\right)$, 
Table 1

Simulation results.

\begin{tabular}{|c|c|c|c|c|c|c|c|}
\hline \multirow[t]{2}{*}{$\bar{\mu}$} & \multirow[t]{2}{*}{$\sigma^{2}$} & \multicolumn{2}{|c|}{$T=30$} & \multicolumn{2}{|c|}{$T=50$} & \multicolumn{2}{|c|}{$T=70$} \\
\hline & & $Q P S_{d}$ & $Q P S_{\delta}$ & $Q P S_{d}$ & $Q P S_{\delta}$ & $Q P S_{d}$ & $Q P S_{\delta}$ \\
\hline 0.5 & 0.5 & 0.30 & 0.25 & 0.29 & 0.23 & 0.28 & 0.23 \\
\hline 0.5 & 1 & 0.29 & 0.25 & 0.29 & 0.24 & 0.29 & 0.24 \\
\hline 0.5 & 2 & 0.29 & 0.25 & 0.30 & 0.25 & 0.29 & 0.24 \\
\hline 1 & 0.5 & 0.23 & 0.21 & 0.18 & 0.19 & 0.14 & 0.17 \\
\hline 1 & 1 & 0.27 & 0.23 & 0.25 & 0.22 & 0.23 & 0.20 \\
\hline 1 & 2 & 0.28 & 0.24 & 0.27 & 0.23 & 0.27 & 0.23 \\
\hline 2 & 0.5 & 0.13 & 0.18 & 0.10 & 0.17 & 0.09 & 0.17 \\
\hline 2 & 1 & 0.18 & 0.19 & 0.12 & 0.17 & 0.09 & 0.17 \\
\hline \multirow[t]{2}{*}{2} & 2 & 0.24 & 0.22 & 0.19 & 0.19 & 0.16 & 0.18 \\
\hline & & \multicolumn{2}{|c|}{$T=100$} & \multicolumn{2}{|c|}{$T=200$} & \multicolumn{2}{|c|}{$T=400$} \\
\hline 0.5 & 0.5 & 0.27 & 0.23 & 0.26 & 0.21 & 0.22 & 0.21 \\
\hline 0.5 & 1 & 0.29 & 0.24 & 0.27 & 0.23 & 0.26 & 0.23 \\
\hline 0.5 & 2 & 0.29 & 0.24 & 0.29 & 0.24 & 0.28 & 0.23 \\
\hline 1 & 0.5 & 0.11 & 0.17 & 0.06 & 0.17 & 0.03 & 0.17 \\
\hline 1 & 1 & 0.21 & 0.19 & 0.16 & 0.17 & 0.08 & 0.16 \\
\hline 1 & 2 & 0.26 & 0.22 & 0.25 & 0.21 & 0.22 & 0.20 \\
\hline 2 & 0.5 & 0.07 & 0.17 & 0.04 & 0.17 & 0.02 & 0.17 \\
\hline 2 & 1 & 0.08 & 0.17 & 0.04 & 0.17 & 0.02 & 0.17 \\
\hline 2 & 2 & 0.12 & 0.17 & 0.06 & 0.17 & 0.03 & 0.17 \\
\hline
\end{tabular}

Note. The table reports the average QPS for the forgetting factor method $(\delta)$ and the Markov-chain method (d). Results are based on 1000 replications.

is the transition probability matrix, and the realization of the sequence at time $t+1$ is defined as

$\widetilde{S}_{a, t+1}=\left\{\begin{array}{l}(1,0)^{\prime}, \text { if } q_{a} \geq \theta \\ (0,1)^{\prime}, \text { otherwise }\end{array}\right.$

where $\theta$ is drawn from a $U[0,1]$. Second, in an analogous way, generate an independent Markovian sequence, $\widetilde{S}_{b, t}$, with its corresponding transition probability matrix, $P_{b}$. Third, generate another Markovian sequence, $\tilde{V}_{t}$, with its corresponding transition probability matrix, $P_{V}$, that governs the changes of synchronisation between $\widetilde{S}_{a, t}$ and $\widetilde{S}_{b, t}$ according to the following rule:

$S_{a, t}=\widetilde{S}_{a, t[1]}$,

$S_{b, t}=\left\{\begin{array}{l}\widetilde{S}_{a, t[1]}, \text { If } V_{t}=(1,0)^{\prime} \\ \widetilde{S}_{b, t[1]}, \text { If } V_{t}=(0,1)^{\prime}\end{array}\right.$,

where $\widetilde{S}_{i, t[1]}$ denotes the first element of the sequence vector for $i=a, b$.

For simplicity, we assume that $p_{a, 11}=p_{b, 11}=0.9$, and $p_{a, 22}=p_{b, 22}=0.8$. Since our application involves dealing with a small sample, we are interested in generating a small number of random changes of synchronisation during the time span, $T$. In doing so, we relate the transition probabilities of the state variable that indicates changes in synchronisation, $p_{v, 11}=p_{v, 22}=p_{v}$, to the sample size, $T$, based on an expected duration of one change over the entire sample, ${ }^{11}$

$p_{v}=1-\frac{2}{T}$.

We control for three dimensions in our simulations. First, we focus on the dispersion between the state-dependent means, defined as $\bar{\mu}=$ $\left|\mu_{S_{i, t}=1}-\mu_{S_{i, t}=0}\right|$, keeping a mean between $\mu_{S_{i, t}=1}$ and $\mu_{S_{i, t}=0}$ equal to zero. We consider a set given by $\overline{\boldsymbol{\mu}}=\{0.5,1,2\}$. Second, we assess the effect of the volatility associated with the innovations of the data. For simplicity, we assume that $\sigma_{11}^{2}=\sigma_{22}^{2}=\sigma_{12}^{2}=\sigma^{2}$, and study different

\footnotetext{
11 We relate the expected duration of a synchronisation regime (high or low), $p_{v}^{E}$, defined as $p_{v}^{E}=\frac{1}{1-p_{v}}$, to the number of time periods that the series would remain in that regime, $\tau$. Since we are interested in generating (on average) one change of synchronisation, $\tau=\frac{T}{2}$.
}

scenarios of volatility given by the set $\boldsymbol{\sigma}^{2}=\{0.5,1,2\}$. Our main interest is in the performance of the models under different sizes of the available sample. Therefore, we evaluate the finite sample properties by repeating the simulations associated with each configuration of parameters for the set of sample sizes, $\mathbf{T}=\{30,50,70,100,200,400\}$. We perform $M=1000$ simulations for each configuration of parameters, $\bar{\mu}$ and $\sigma^{2}$, and for each sample size, $T$, under consideration.

\subsubsection{Results}

At each $m$-th replication, we compute the time-varying synchronisation estimated with the forgetting factor $(F F)$ method proposed in this paper and define it as $\delta_{t}^{m}$. We also compute the time-varying synchronisation estimated with the Markov-chain $(M C)$ method, proposed in Leiva-Leon (2016), and define it as $d_{t}^{m}$. For each replication, we compute the Quadratic Probability Score (QPS) associated with each method, taking the variable indicating the true synchronisation changes, $V_{t}=\tilde{V}_{t[1]}$, as our reference. Our object of interest is the average QPS over the $M$ replications associated with each method, calculated as,

$$
\begin{aligned}
& Q P S_{\delta}=\frac{1}{M} \sum_{m=1}^{M}\left[\frac{1}{T} \sum_{t=1}^{T}\left(V_{t}^{m}-\delta_{t}^{m}\right)^{2}\right], \\
& Q P S_{d}=\frac{1}{M} \sum_{m=1}^{M}\left[\frac{1}{T} \sum_{t=1}^{T}\left(V_{t}^{m}-d_{t}^{m}\right)^{2}\right] .
\end{aligned}
$$

Table 1 reports the simulation results based on $M=1000$ replications. The results indicate that the ability of both methods to accurately infer changes in synchronisation increases as the dispersion between the state-dependent increases and the volatility of the innovations decreases. Regarding the scenarios of different sample sizes, the table shows that, for small samples $(T=30)$, the $F F$ method outperforms the $M C$ method in $78 \%$ of the cases. However, when the sample increases, the relative performance of the $F F$ method with respect to the $M C$ method tends to decrease. For $T=50, T=100$, and $T=200$, the FF method outperforms the $M C$ method in $67 \%, 56 \%$, and $44 \%$, of the cases, respectively. From these exercises we conclude that the method to assess changes in synchronisation proposed in this paper performs significantly better than the $M C$ method in small samples, but it also is competitive in large samples. 


\section{Assessing changes in regional interdependence}

\subsection{Data}

The sample consists of 213 regions, following the NUTS- 2 classification, that correspond to 16 European countries: the EU-12 (Austria (AT), Belgium (BE), Finland (FI), France (FR), Germany (DE), Ireland (IE), Italy (IT), Luxembourg (LU), Netherlands (NL), Portugal (PT), Spain (ES) and Greece (GR)) and Denmark (DK), Norway (NO), Sweden (SE) and the United Kingdom (UK). For Germany, data of the eastern Landers and Berlin are not available prior to 1991. Therefore, they are not included in our analysis.

The availability of regional data on a high frequency basis and for a long span is scarce. To analyse the synchronisation of the regional business cycles, we employ the most comprehensive measure of real economic activity, that is, annual real GDP data, as quarterly data is not available. It has to be acknowledged that annual data are less noisy than quarterly ones, so they could be even more reliable to establish robust facts on real economic activity in spite of the loss of information on short-term dynamics (see Giannone et al. (2010)). Moreover, to capture a complete business cycle several years are needed. ${ }^{12}$

GDP is the most common measure of economic activity in the literature on business cycle synchronisation at country level while the measure of economic activity at regional level varies across studies, as previously mentioned. The series cover a period of 35 years, from 1980 to 2014. Thus, the potential effects of the Great Recession and the European Debt Crisis on the regional business cycle of the European countries are analysed for the first time.

The source of the data is the Cambridge Econometrics database, which contains data collected from Eurostat's REGIO database and from AMECO, a dataset provided by the European Commission's Directorate General Economic and Financial Affairs (DG EcFin). ${ }^{13}$

\subsection{Bilateral economic linkages}

Much of the literature about business cycle synchronisation is based on dynamic factor models. These models try to disentangle between common and idiosyncratic components in order to infer changes in the relationship between the real activity of different economies and some common factors. Some examples are Kose et al. (2012), Del Negro and Otrok (2008) and Lee (2013), among others, at country level and Marino (2013) at regional level. However, since factor models typically assume that the idiosyncratic components are independent from each other, they are not able to provide information about changes in the bilateral relationship of the business cycles associated with a pair of regions. Our approach would provide a more detailed picture of the regional business cycle linkages and would allow us to identify the regions acting as main conduits in the propagation of business cycle shocks.

In order to illustrate how to interpret the output of our econometric framework, Fig. 1 shows the time-varying business cycle synchronisation for a couple of selected pairs of regions. Chart A illustrates the synchronisation between two capital regions of relatively small countries, Brussels-Capital (BE) and Noord-Holland (NL). The chart shows an almost constant level of synchronisation until the early 1990s. However, after the Maastricht Treaty, both regions experienced a persistent increase in business cycle synchronisation until the end of the sample, in 2014. Chart B depicts the pattern of synchronisation between two capital regions of relatively large countries, Ile de France (FR) and Inner

\footnotetext{
12 Bandres et al. (2017) and Gadea-Rivas et al. (2018), using the same dataset, are able to properly date the business cycle dynamics of different groups of European regions.

13 The GDP series are deflated with 2005 constant price euros using price deflators obtained from AMECO.
}

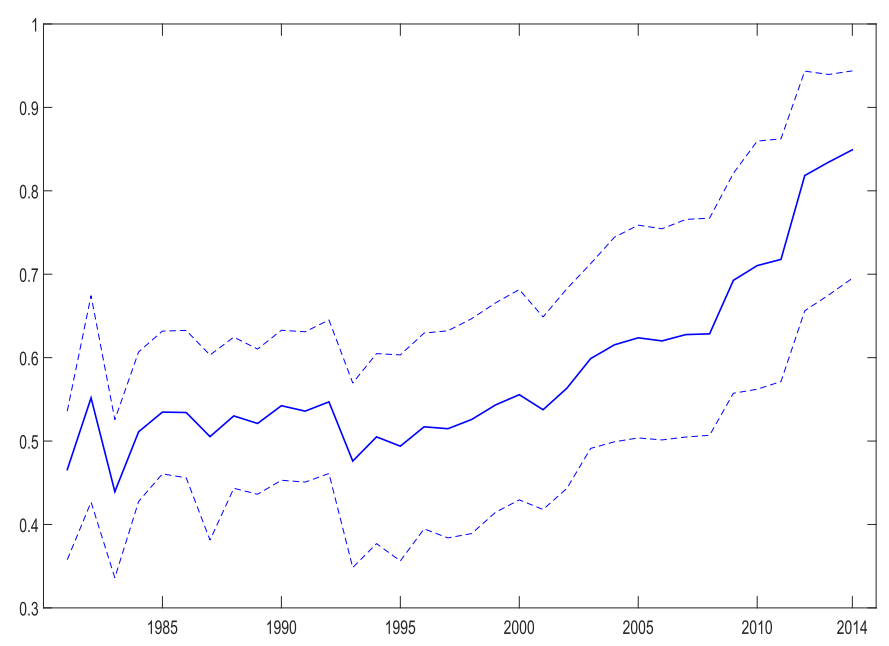

(a) Brussels-Capital vs. Noord-Holland

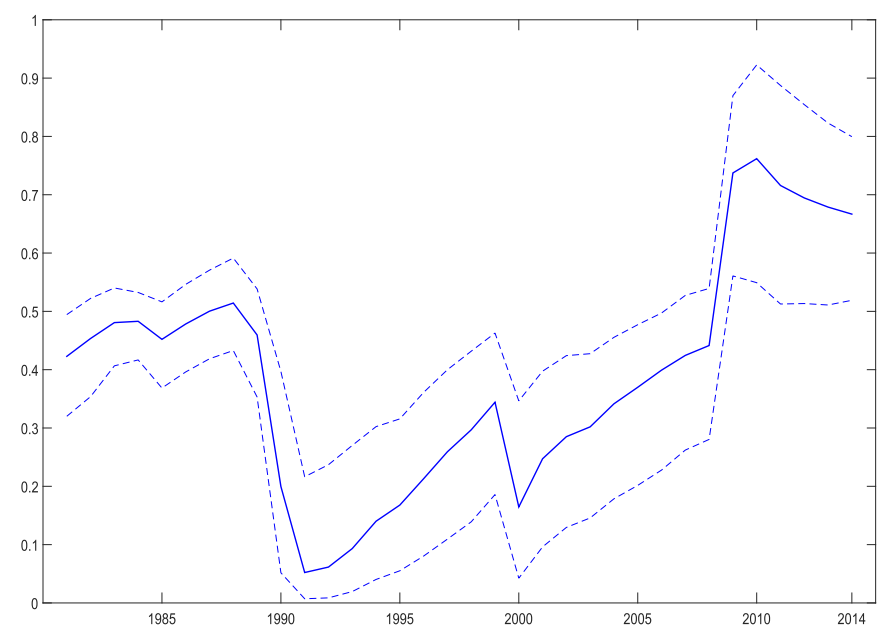

(b) Ile de France vs. Inner London

Fig. 1. Time-varying regional business cycle synchronisation. Note. The figure plots the time-varying synchronisation between pairs of European regions. Solid lines represent the 0.5 quantile and dashed lines represent the 0.75 and 0.25 quantiles of the distribution of $\delta_{t}^{a b}$.

London (UK). The chart shows an upward trend in synchronisation after the Maastricht Treaty in 1992, followed by a transient fall in 2000 due to the introduction of the euro in only one of the two economies, and a significant increase in the level of synchronisation after the Great Recession, which remained quite high a few years after it.

In addition, we can adapt the econometric framework proposed in this paper to identify those European regions more affected by the "Brexit", that is, the withdrawal of the United Kingdom (UK) from the European Union (EU). See Appendix B.

\subsection{Overall regional synchronisation}

To investigate the overall spectrum of the aggregate economic interlinkages between the European regions, we estimate the model in equations (1)-(7) for each pair of regions in our sample. Since our sample consists of 213 regions, there are a total of 22,578 pairwise possible combinations. It is important to mention that, in spite of certain common patterns, there is a significant heterogeneity associated with the idiosyncrasy of each bilateral regional relationship, which may be due to multiple factors. The aim of this section is not to investigate the 
characteristics behind each pairwise synchronisation, but to identify the main features of their overall patterns that would help us to understand the propagation of business cycle shocks at the regional level.

We start by providing a general assessment about the regions experiencing the highest levels of synchronisation with the rest of Europe. To do so, we compute the following index of "aggregate" synchronisation for each region:

$d_{t}^{i}=\frac{1}{n} \sum_{j=1}^{n} \delta_{t}^{i j}$, for $i=1,2, \ldots, n$,

where $n=213$ regions and $d_{t}^{i}$ provides information about the overall degree of synchronisation of region $i$ with the rest of Europe at time $t$. Fig. 2 plots heat maps of the synchronisation patterns in Europe for different time periods, measured by $d_{t}^{i}$, namely, for 1981 (the beginning of our sample), 1999 (the introduction of the euro), 2009 (the Great Recession) and 2014 (end of the sample). Chart A of Fig. 2 shows that, in 1981, almost all the regions of Europe were experiencing a relatively low synchronisation, ranging between 0.3 and 0.5 . However, during the implementation of the euro, many regions of Spain, France, Germany and Italy, and even some regions of the United Kingdom, became more synchronised with the rest of Europe, exhibiting levels of synchronisation between 0.5 and 0.7 , as can be seen in Chart B. In the middle of the Great Recession, many regions experienced high levels of synchronisation, showing evidence of the propagation of a contractionary shock during this time in particular, as can be seen in Chart C. For example, most of the Spanish regions experienced synchronisation levels with the rest of Europe of between 0.7 and 0.9. This agrees with the fact that Spain was one of the European countries most affected by the Great Recession. Finally, Chart D plots this information at the end of the sample, 2014, showing that although the overall degree of regional synchronisation has suffered a certain setback after the end of the Great Recession, it has remained at relatively high levels, ranging between 0.5 and 0.7 .

Having estimated all the economic bilateral linkages between the European regions, $\delta_{t}^{a b}$ for all $i \neq j$, we now analyse this information from a time dimension. Croux et al. (2001) investigate synchronisation between European countries at the frequency domain by measuring their cohesion. Cohesion measures are defined as indexes that summarize information about bilateral relations and that are useful to provide an overall assessment of the interrelations between a set of elements (markets, countries, etc.). We compute indexes of cohesion to measure the overall degree of European synchronisation as in Croux et al. (2001). Nevertheless, our analysis differs from theirs in two important features. First, we focus on the time rather than the frequency domain and, second, we are interested in measuring cohesion based on regional rather than national disaggregation. Accordingly, our proposed timevarying European cohesion measures are defined as follows,

$c_{t}^{\text {europe }}=\frac{\sum_{a \neq b} \omega_{a, t} \omega_{b, t} \delta_{t}^{a b}}{\sum_{a \neq b} \omega_{a, t} \omega_{b, t}}$, for $a, b=1,2, \ldots, n$

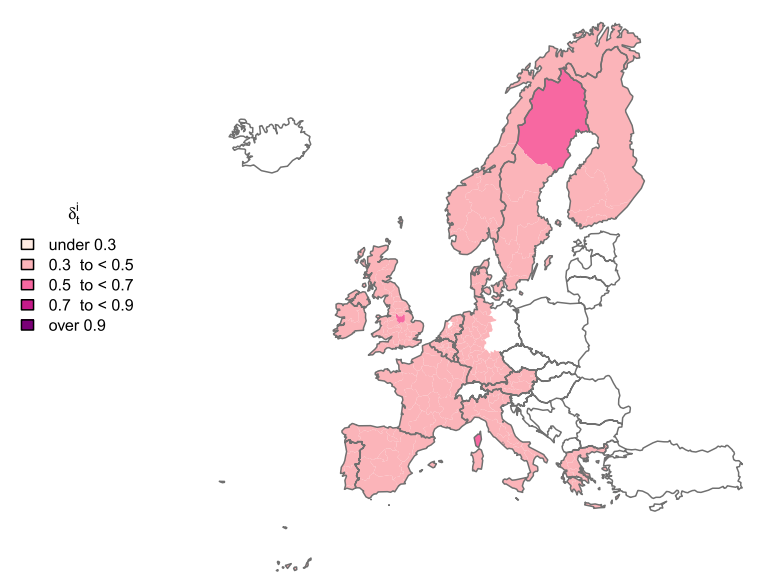

(a) 1981

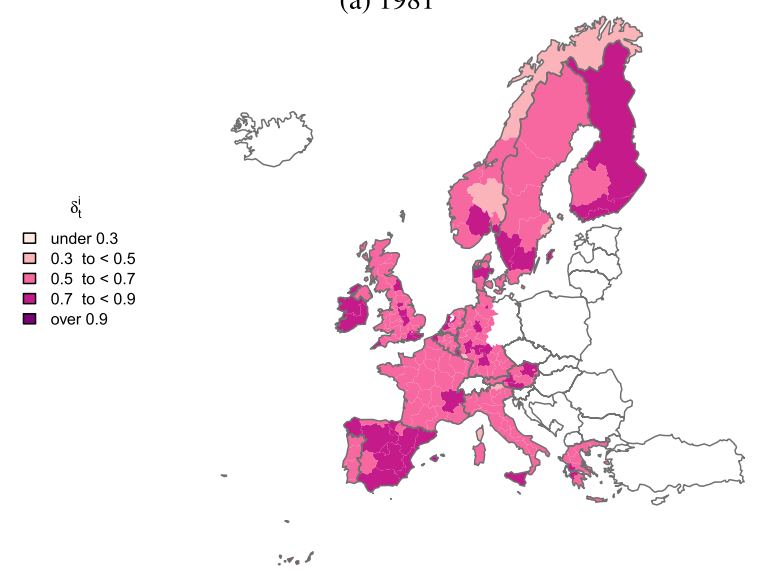

(c) 2009

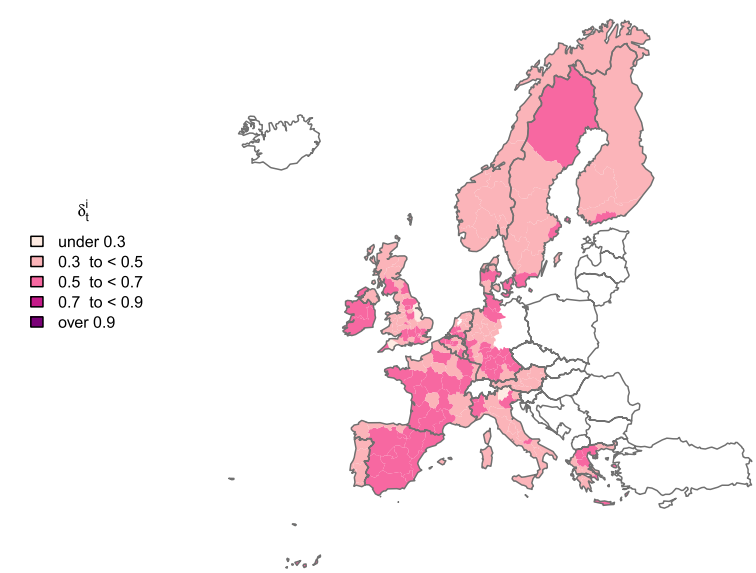

(b) 1999

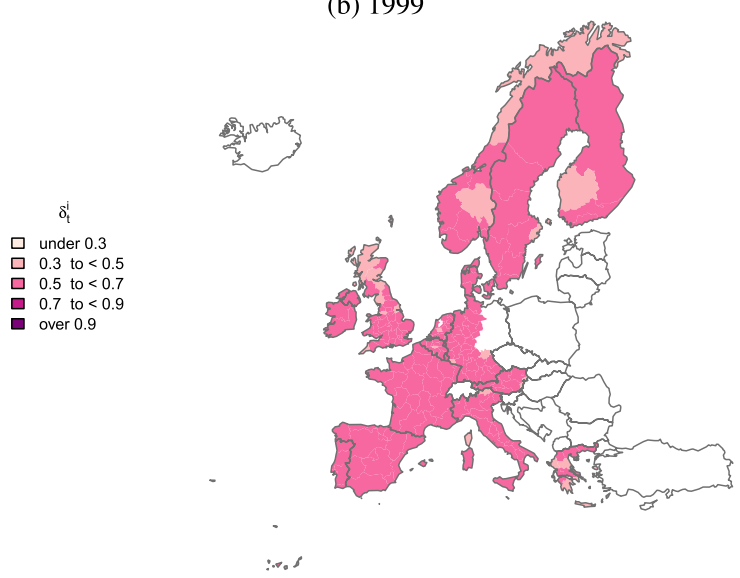

(d) 2014

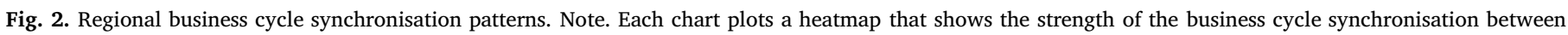

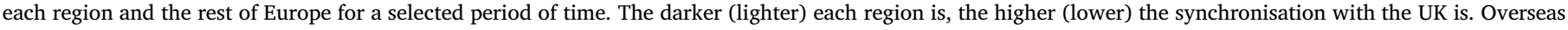
regions of France and Portugal are not displayed because of the dimensions of the map. 


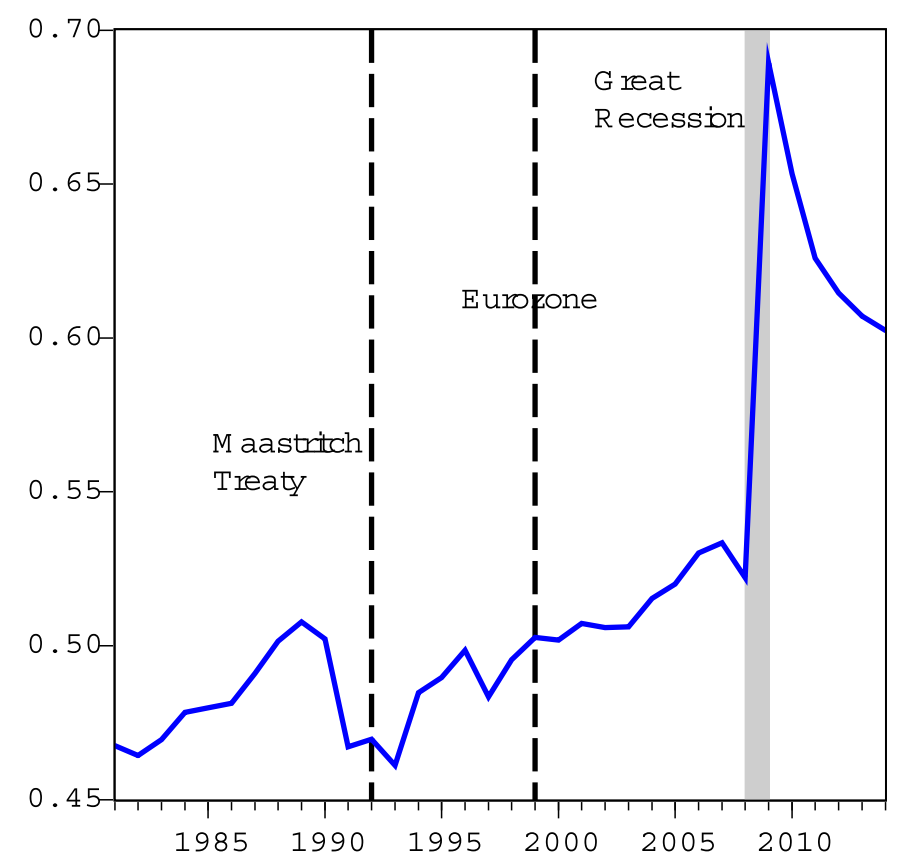

Fig. 3. Time-varying European cohesion. Note. The figure plots the European cohesion measure over time. Vertical lines and bars refer to specific events.

where, $\omega_{a, t}$ and $\omega_{b, t}$ denote the weights that regions " $a$ " and " $b$ "receive, respectively, defined as the GDP share of each region with respect to the total GDP in Europe.

Fig. 3 displays the time-varying European business cycle cohesion. It shows that, during the 1980s, European cohesion exhibited an upward trend. This increasing cohesion pattern is associated with the signing of the Single European Act in 1986, a treaty which forms the basis for a comprehensive program aimed at eliminating obstacles to the free movement of goods across EU borders and thus giving rise to the "single market". However, the index of European cohesion experiences a significant drop in the late 1980s. This could be associated, on the one hand, to the fall of Berlin Wall in 1989 and, on the other hand, to the early 1990s global recession. Both events lead to an increasing heterogeneity of output fluctuations across European regions around that time. ${ }^{14}$

Nevertheless, since the Maastricht Treaty was signed in 1992, European cohesion again exhibited an upward trend that continued after the introduction of the euro, in 1999, until 2007. ${ }^{15}$ Notice that European cohesion increased by about 5 basis points (from 0.47 to 0.52 ) between 1981 and 2007. This increase may be partially attributed to the process of European unification in institutional and economic terms. However, between 2008 and 2009 the cohesion increased dramatically by 17 basis points (from 0.52 to 0.69 ), reaching its maximum level in our sample. This leap in the cohesion was obviously influenced by the Great Recession, since contractionary business cycle shocks were propagated through most European regions. ${ }^{16}$ These results imply that neither the

\footnotetext{
14 The fall of Berlin Wall in 1989, which resulted in an important economic shock to Germany and caused a cyclical asymmetry with the rest of Europe, could also have increased heterogeneity in the transmission of business cycle shocks across European regions. In particular, the subsequent reunification process triggered an expansion and disequilibria in the German economy at a time when the main economies in the EU were decelerating.

15 Based on a common factor approach, Lee (2012, 2013) find an increase in output synchronisation across European countries during the run-up to the inception of the euro that did not continue afterwards.

${ }^{16}$ In this line, Bandres et al. (2017) compute the rolling average of spatial correlation using Moran's modified statistic for European regions. They observe that spatial correlation progressively increased during the convergence process and rocketed during the Great Recession.
}

Maastricht treaty nor the introduction of the euro managed to synchronise European regions in decades as much as the Great Recession did in only two years. ${ }^{17}$ Moreover, despite the fact that the cohesion index fell after the end of the Great Recession, it remained at relatively high levels until the end of our sample, in 2014, suggesting that the Great Recession had an ex-post significant impact on the regional business cycle synchronisation of Europe. ${ }^{18}$

Additionally, we can also compute cohesion measures to analyse the synchronisation degree by country. See Appendix C.

\subsection{The role of the economic size of regions}

In previous sections, we have analysed the synchronisation of regional GDP growth assigning the same importance to all the regions in Europe and, consequently, treating all bilateral relationships in the same way. However, some regions may play a more important role in the propagation of business cycle shocks due to their size in economic terms, i.e. their GDP share with respect to the total GDP of Europe. For example, two highly synchronised regions that also have a large economic size would act as channels in the propagation of business cycle shocks more prominently than (i) two highly synchronised regions of small economic size, or (ii) two regions of large economic size but unsynchronised. Therefore, in this section, we focus on identifying the bilateral cyclical relationships between regions that are (i) highly synchronised and (ii) large in economic size.

To identify the linkages of regions depending on their economic size, we construct a weighted measure of synchronisation based on the same notion of cohesion described in Equation (42). As a consequence, our modified synchronisation measure is given by

$\widetilde{\delta}_{t}^{a b}=\frac{\left(\omega_{a, t} \omega_{b, t} \delta_{t}^{a b}\right)-\delta^{\min }}{\delta^{\max }-\delta^{\min }}$,

where $\delta^{\min }$ and $\delta^{\max }$ are coefficients used to normalize $\widetilde{\delta}_{t}^{a b}$ between 0 and 1 for easier interpretation and denote the minimum and maximum value of the term $\left(\omega_{a, t} \omega_{b, t} \delta_{t}^{a b}\right)$ for all $a \neq b$, and for $t=1,2, \ldots, T$, respectively. Accordingly, pairs of regions with high values of $\widetilde{\delta}_{t}^{a b}$ are "the most central regions", those that are more connected to the rest of regions taking into account both economic size and degree of synchronisation. Thus, these pairs could be interpreted as the most prominent relationships for the transmission of business cycle shocks.

The upper left-hand chart of Fig. 4 plots the main linkages between European regions with a weighted synchronisation higher than 0.25 for 1981. We have also assessed the results with different thresholds, but the main conclusions remain unchanged. ${ }^{19}$ This set of linkages can be viewed as a European business cycle network that has a star-shaped topological structure, in which the central region is Ile de France and the arrows around it are Inner London, some regions located in West Germany and other regions in Northern and Central Italy. It is interesting to note that the interconnections identified by our proposed weighted synchronisation measure correspond very closely to the historical trade routes in Europe, located mainly in Northern Italy, the Netherlands and Ile de France. The latter used to play an important role as an articulator. ${ }^{20}$ The upper right-hand chart of Fig. 4 plots the main

\footnotetext{
17 This result is in line with that of Canova et al. (2012), who show that time variations in the features and the transmission of cyclical fluctuations across countries appear to be linked more to the general process of European convergence taking place since the mid-80s than to institutional changes.

18 Ciccarelli et al. (2016) find that, while the Great Recession features the largest real and financial shocks, their spillovers to advanced economies were similar to those observed during previous recessions.

19 Bailey et al. (2016) propose a procedure for selecting significant bilateral static correlations, computed using the entire sample. However, since our approach produces measures that can be interpreted as time-varying correlations, this selection procedure is not suitable for our case.

20 See Braudel (1979) for a detailed description.
} 

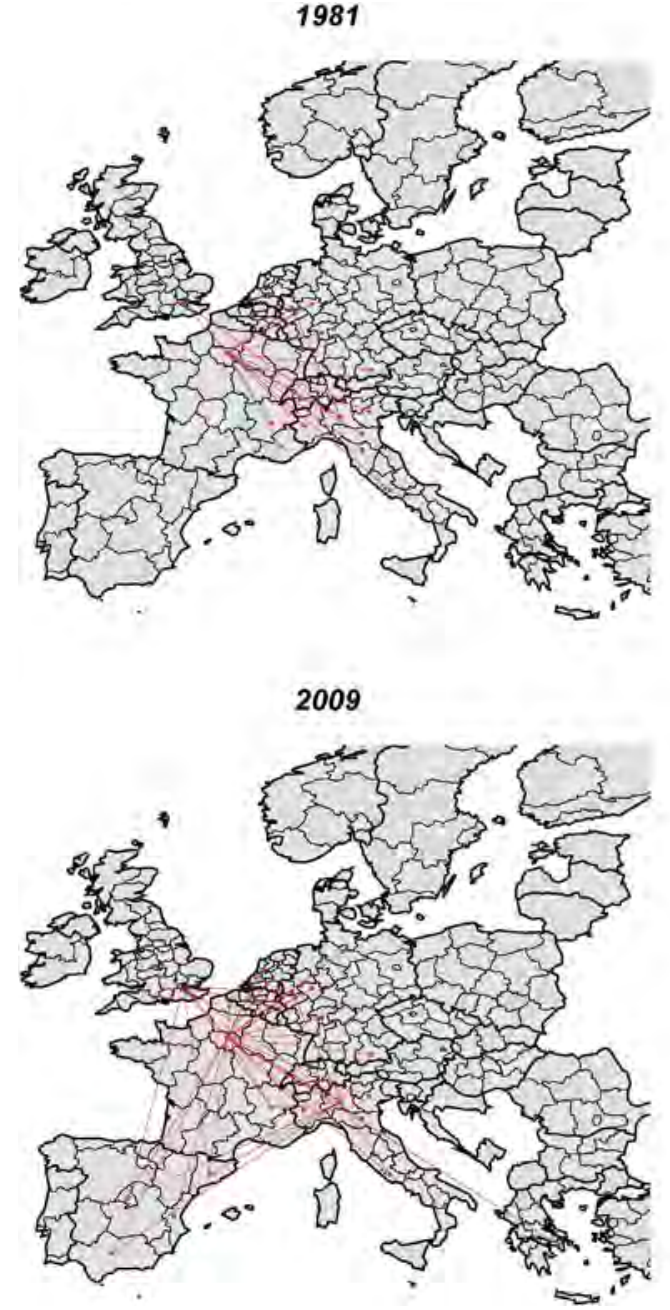

1999

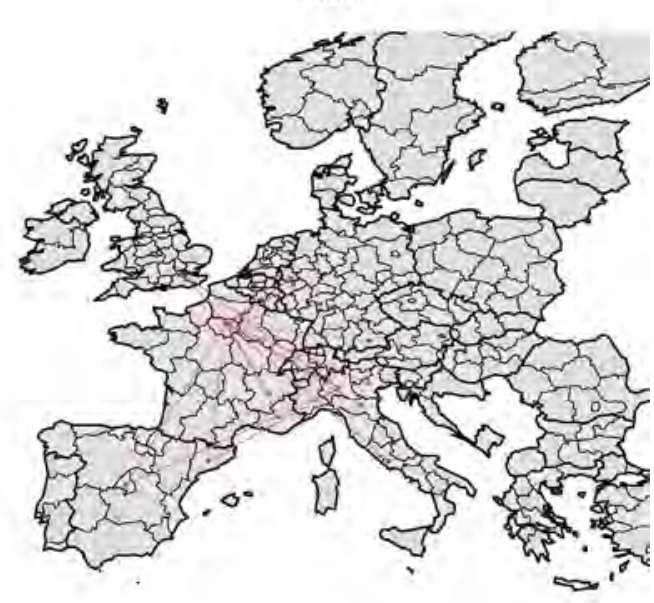

2014

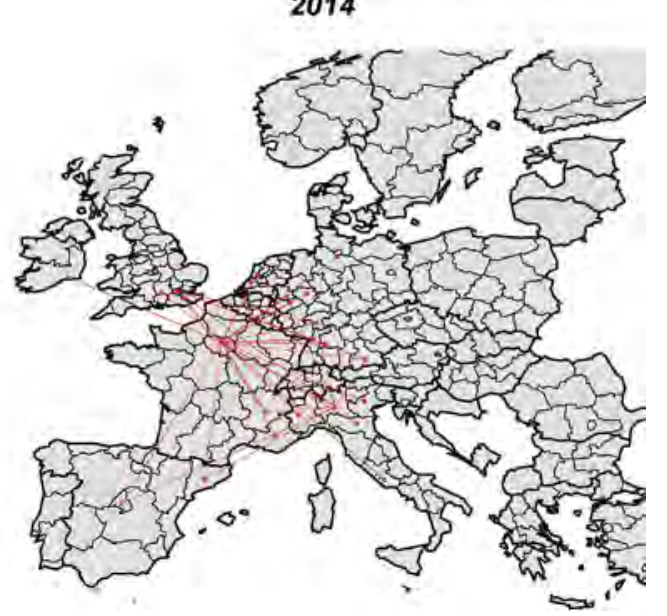

Fig. 4. Main European regional economic linkages. Note. A red line connecting two regions indicates a weighted synchronisation higher than or equal to 0.25 between them. (For interpretation of the references to colour in this figure legend, the reader is referred to the Web version of this article.)

linkages for 1999, when the euro was introduced. The chart shows a topological structure similar to that of 1981, the main difference being that, during this period, some Spanish regions became connected to Ile de France and Lombardia. These results imply that the implementation of the euro made Spain which, at that time, was following a prolonged expansionary path, a significant contributor to European business cycle dynamics. ${ }^{21}$ The lower left-hand chart of Fig. 4 plots the main linkages for 2009, in the middle of the Great Recession. The chart shows a significantly more connected topological structure of the European business cycle network. The number of connections associated with Ile de France, Inner London and Lombardia increased notably. Some Spanish regions became connected to French, Italian and English regions, and the capital region of Greece became connected to the network. Accordingly, this chart shows which regions were the most prominent in propagating contractionary shocks in Europe during the Great Recession. Finally, the lower right-hand chart of Fig. 4 plots the main linkages for 2014, the end of our sample. We observe that regional linkages have not changed significantly after the Great Recession, with the sole exceptions of the disconnection of Greece and the lower connectivity of some Spanish regions. Overall, these results show that Ile de France is acting as the most central region in the propagation of business cycle shocks

\footnotetext{
${ }^{21}$ Notice that, during this period, the relative weight of the Spanish regions remained relatively stable, indicating an increased synchronisation as the source of these linkages.
}

in Europe, followed by Inner London and Lombardia.

\section{Synchronisation and sectoral composition}

In previous sections, we have studied the synchronisation of the European regions and have assessed the role of the economic size of regions in characterizing the propagation of business cycle shocks. In this section, we examine the role of other factors in explaining the evolution of the synchronisation between regions, paying particular attention to the similarities in their productive structure. Sectoral composition could play a significant role in explaining synchronisation since it may be viewed as a proxy for the transmission of shocks between regions. It is worth noting that we are not investigating a causal relationship between sectoral composition and synchronisation, but looking for the degree of association between them.

The literature on synchronisation has mainly focused on analysing the role of sectoral composition in determining business cycle synchronisation at country level. ${ }^{22}$ However, studies that investigate the extent

\footnotetext{
22 Clark and van Wincoop (2001) examine several measures of sectoral dissimilarity. They find that these measures explain, to some extent, the low crosscountry correlation of employment between the US and the EU, but they find no correlation with the GDP. Imbs (2004) computes a specialization index of industries and identifies a low business cycle correlation between highly specialized regions.
} 


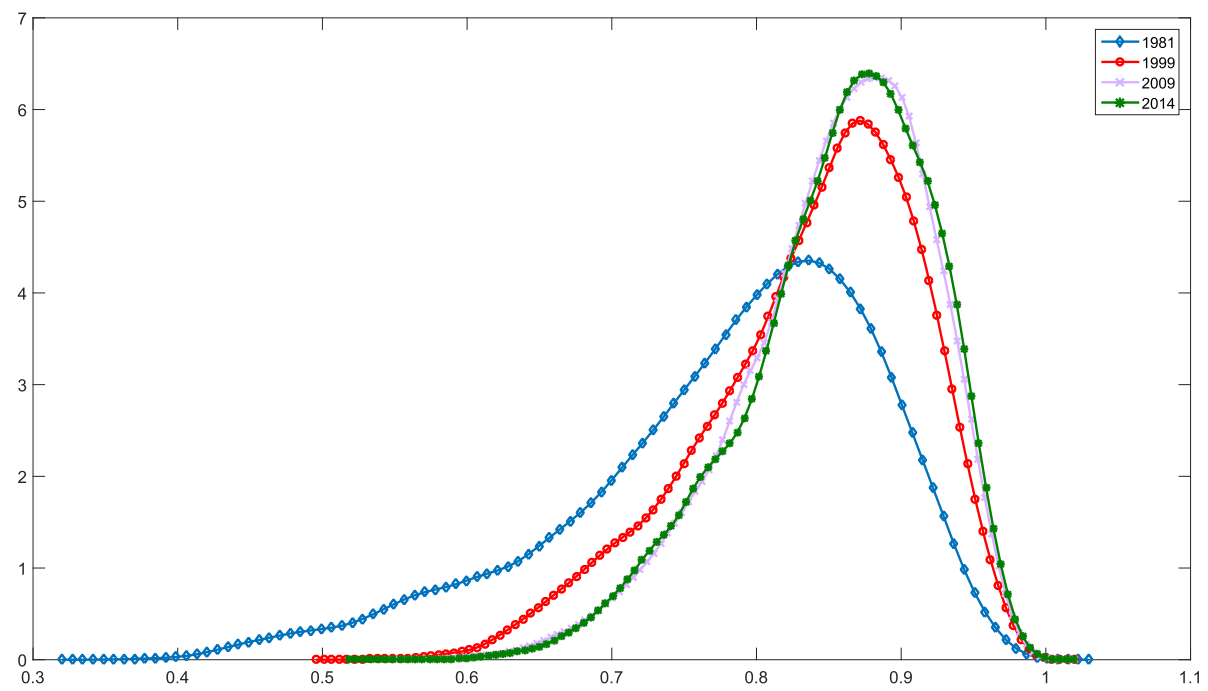

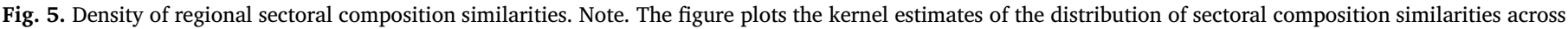
the European regions for selected periods. Sectoral similarity is measured following Imbs (2004).

to which sectoral patterns can explain regional synchronisation are quite scarce and their findings are not conclusive. For example, Barrios et al. (2003) compute industrial similarity using a sample of eleven UK regions and six euro area countries for the period 1966-1997 and find that industrial similarity does not explain the decline in the UK-EU business cycle correlations. Belke and Heine (2006) test the impact of industrial structure on the regional employment cycles of thirty European regions for the period 1975-1996, finding that differences in industry structure account for the decline in employment synchronicity among regions. ${ }^{23}$ Apart from their different datasets (variables used and temporal and spatial dimensions considered), since these studies are based on simple linear regression approaches, plausible reasons for the differences in their results could be the existence of a nonlinear relationship between sectoral composition similarities and business cycle synchronisation, or a potential instability in this relationship over time, or both. In this section, we tackle these possibilities parsimoniously to provide a robust assessment of the relationship between sectoral composition and business cycle synchronisation at the regional level.

\subsection{Similarities in sectoral composition}

First, we quantify the degree of similarity in the bilateral sectoral composition of European regions. We compute the variable sectoral, which captures the similitude between the productive structures of the regions. We adapt the expression used by Imbs (2004) and measure sectoral composition similarity as follows,

$C_{t}^{a b}=1-\frac{\sum_{i=1}^{k}\left|c_{t}^{i, a}-c_{t}^{i, b}\right|}{2}$,

where $c_{t}^{i, j}$ is the employment share of sector $i$ in region $j$ at time $t$ and $k$ the number of sectors. Given the available data, we consider 6 sectors: Agriculture, Industry, Construction, Financial and business services (FBS), Non-market services, and a sector involving the following categories; Wholesale, retail, transport, accommodation, food services, information and communication (WRTAFIC). The index of sectoral composition similarities, $C_{t}^{a, b}$, ranges from 0 , when the sectoral structures of regions $a$ and $b$ are completely different, to 1 , when the sectoral structures are identical.

\footnotetext{
23 Barrios and De Lucio (2003) show that the more similar the sectoral structures of Spanish and Portuguese regions (1975-1998) are, the more correlated the employment cycles are.
}

Fig. 5 displays the estimated density of regional sectoral composition similarities for selected years, $\mathbf{C}_{t}=\left\{C_{t}^{a, b}: \forall a \neq b\right\}$. There is a displacement of the kernel density mass towards the right tail, which indicates an increase in sectoral similarity over time. Moreover, this has been a gradual but persistent pattern over the entire sample period (1980-2014). ${ }^{24}$ The data on sectoral composition shows that, at the beginning of the sample, in 1980, there was substantial heterogeneity in sectoral composition similarities across Europe. However, at the end of the sample, in 2014, there is a more homogeneous pattern with most of the regions exhibiting high levels of sectoral composition similarities, as can be seen in the heat maps of Fig. 11 in Appendix D. ${ }^{25}$ This result has important implications for the propagation of shocks throughout the European economy, since the more similar the economic structure of regions is, the more similar their responsiveness to shocks would be, potentially amplifying their effects at the aggregate level.

\subsection{Parametric regression analysis}

We analyse the relationship between synchronisation and sectoral composition by controlling for additional potential determinants of

Table 2

Estimation of panel.

\begin{tabular}{lll}
\hline variables & coeff & t-ratio \\
\hline constant & 0.3726 & 113.5594 \\
emu & -0.0026 & -3.3216 \\
groups & -0.0013 & -1.6257 \\
country & 0.0451 & 33.4649 \\
size-regions & 0.9623 & 22.6448 \\
size-countries & 0.0195 & 5.6318 \\
sectoral & 0.1142 & 30.8652 \\
\hline
\end{tabular}

Note. Standard errors are HAC robust and fixed time effects are included.

\footnotetext{
${ }^{24}$ The associated figures for all the years in the sample are not reported in the paper to save space, but they are available upon request.

${ }^{25}$ The heat maps offer richer information if one is interested in analysing a particular set of regions. For instance, in the map of 1980, it is possible to identify cold areas that correspond to Greek regions that report degrees of sectoral similarity around 0.3 . Intermediate maps, which are not presented to save space, and the details by region are available upon request.
} 

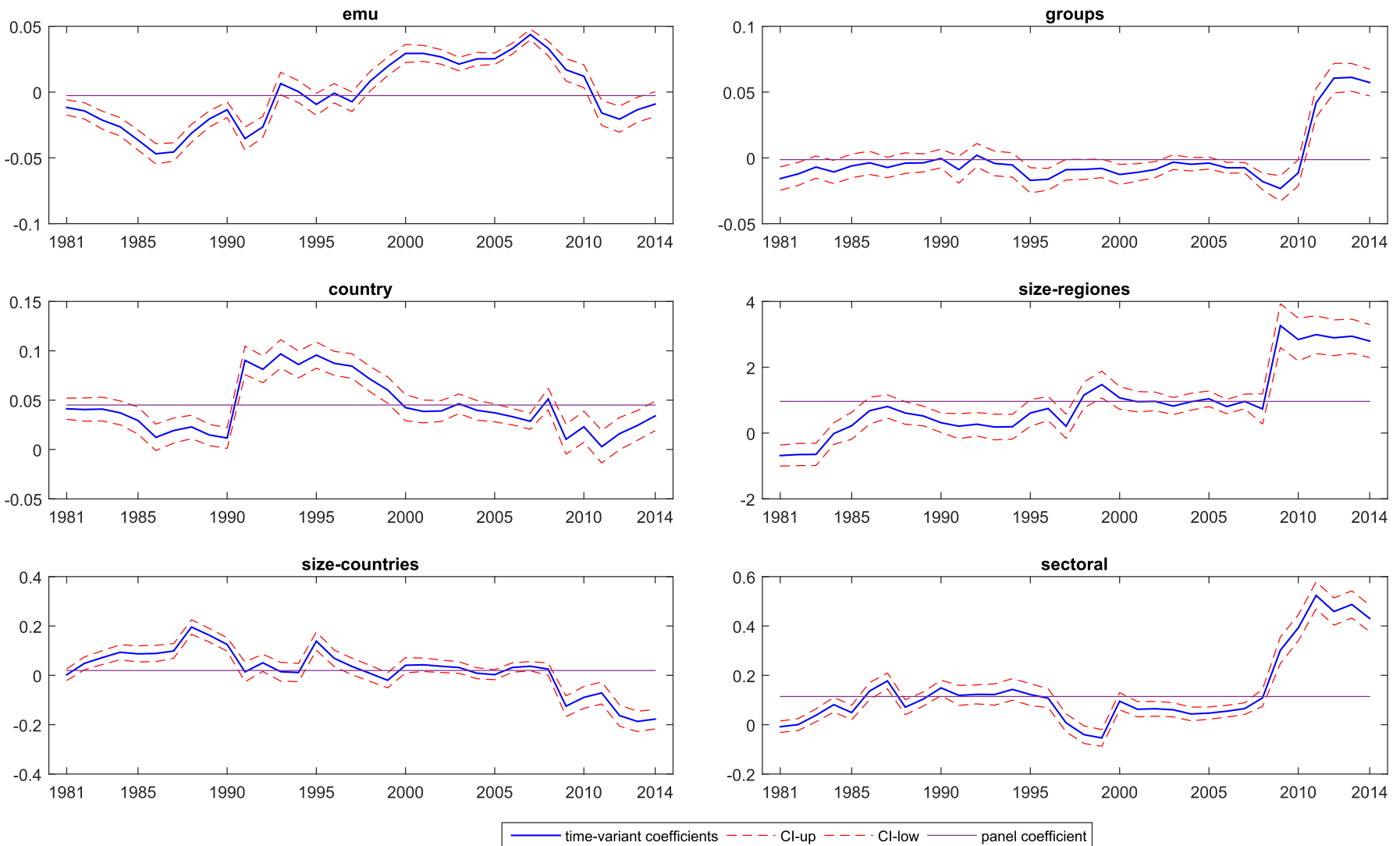

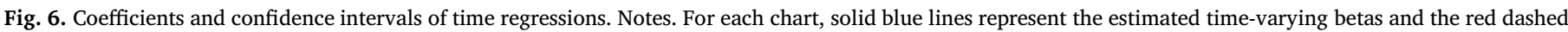

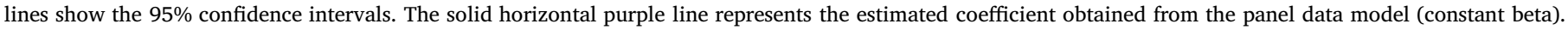
(For interpretation of the references to colour in this figure legend, the reader is referred to the Web version of this article.)

regional synchronisation. To do so, we model the original synchronisation measures, $\delta_{t}^{a b}$, as a function of sectoral composition, $C_{t}^{a b}$, and a set of other regressors. These regressors can be sorted into two groups. The first group corresponds to institutional and geographical factors:

- EMU : defined as a dummy that takes value 1 if both regions belong to the EMU and 0 otherwise.

- Group : defined as a dummy that takes value 1 if both regions belong to the same group of countries and 0 otherwise; the groups are defined as "Central countries" (BE, DE, FR, NL, LU, AT), "Nordic countries" (DK, SE, NO, FI), "Mediterranean countries" (GR, IT, PT, ES), "British Isles" (IE, UK).

- Country : defined as a dummy that takes value 1 if both regions belong to the same country and 0 otherwise.

The second group is related to the economic importance of the regions, proxied by their economic size:

- Size - reg : defined as the economic weight of each pair of regions: $\frac{Y_{a, t}+Y_{b, t}}{\sum_{i=1}^{n} Y_{i, t}}$, where $Y_{i}$ is the real GDP of region $i$ and $n$ is the total number of regions.

- Size - country : defined as the economic weight of the countries to which each pair of regions belong: $\frac{z_{a, t}+Z_{b, t}}{\sum_{i=1}^{\eta} Z_{i, t}}$ where $Z_{i}$ is the real GDP of the country to which region $i$ belongs and $\eta$ is the number of countries.

Notice that the first three variables are dummies that represent geographical and institutional characteristics so they have no time dimension. However, the remaining three variables are time-varying. ${ }^{26}$

In order to account for the heterogeneity in the cross-sectional dimension and over time, we estimate a panel regression with fixed time effects. ${ }^{27}$

$\delta_{t}^{a b}=\beta X_{t}^{a b}+\tau_{t}+\varepsilon_{t}^{a b}$,

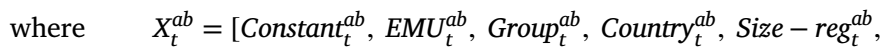
Size - country ${ }_{t}^{a b}$, Sectoral $\left.{ }_{t}^{a b}\right] .{ }^{28}$ The estimation results are reported in Table 2 and indicate that most of the explanatory factors have a positive and statistically significant relationship with the regional synchronisation. In particular, Size $-r^{a} g_{t}^{a b}$ and Sectoral $l_{t}^{a b}$ are the factors with the largest effect on synchronisation. The exceptions are the $E M U_{t}^{a b}$ and Groups ${ }_{t}^{a b}$ that have a negative relationship with synchronisation, although the magnitude of the associated coefficients is almost

\footnotetext{
${ }^{26}$ We have calculated the covariance matrix in order to discard multicolinearity problems. We find that correlations are low, except in the cases of Group and Country (0.50), Groups and EMU (0.41) and Size - country and Size - reg (0.29).

${ }^{27}$ We have tested for the presence of unit roots in the time-varying variables, synchronisation and the sectoral index. The Augmented Dickey-Fuller test (Dickey and Fuller, 1979) and the Mz test of Ng and Perron (2001) do not reject the null hypothesis in almost all cases. Furthermore, we can accept the existence of cointegration between time-varying variables using the test of Phillips and Moon (1999). In any case, Phillips and Moon (1999) show that the fact that $n>>t$, as in our case, guarantees the consistency of our estimates.

${ }^{28}$ We acknowledge that there could be other variables that explain synchronisation, but we are interested in understanding possible associations, not causal relationships.
} 
negligible and not statistically significant for the case of $\operatorname{Groups}_{t}^{a b}$. Therefore, this preliminary analysis indicates that, after controlling for other factors, sectoral composition has a significant effect in explaining changes in synchronisation.

To investigate potential instabilities over time in the relationship between regional synchronisation and its driving factors, we estimate a cross-sectional regression for each time period (year) in the sample.

$\delta^{a b \mid t}=\beta_{t} X^{a b \mid t}+\varepsilon^{a b \mid t}$.

Accordingly, we run $T$ regressions using OLS and obtain the associated time-varying coefficients, $\beta_{t}$, for $t=1,2, \ldots, T$. The dynamics of the estimated regression coefficients for all the years are displayed in Fig. 6 . The results indicate that the estimated dynamic coefficients, $\widehat{\beta_{t}}$, in general, fluctuate closely around the constant estimates from the panel regression, $\hat{\beta}$, implying that the relationship between synchronisation and its explanatory factors has remained broadly stable over time. However, there are some important exceptions, especially since the Great Recession.

Of the institutional factors, EMU membership (emu) has a positive and significant effect on regional synchronisation that begins in the late 1990s with the introduction of the euro, remaining high until the arrival of the Great Recession. The effect then became negative since it affected the eurozone countries with different intensities. Belonging to the same group of countries (group) does not explain, in general, regional synchronisation since its coefficient is hardly ever significant, except after the Great Recession, when the effect is significantly positive. This implies that the arrival of the Great Recession triggered a stronger synchronisation among regions of the same group of countries. The effect of the variable country is positive and significant only during the period between the Maastricht Treaty and the introduction of the euro, pointing to a strong intra-country synchronisation during that time. $^{29}$

As for economic factors, their corresponding relationships with regional synchronisation have remained relatively stable over time, with the exception of the last part of the sample (Fig. 6). The drop in the coefficient for the EMU is consistent with Lee's (2012,2013) finding of a decreasing importance of the European regional factors.

Moreover, after the Great Recession, the correlation between synchronisation and two economic factors, size-reg and sectoral, significantly increased. These results indicate that, taking into account additional factors, the similarities of regional sectoral composition have become significantly more important in explaining regional synchronisation in Europe since the Great Recession.

\subsection{Nonparametric regression analysis}

Previous studies have used parametric econometric approaches, similar to those described in Section 4.2, to evaluate the relationship between similarities in sectoral composition across countries and international business cycle synchronisation (Imbs (2004) and Ductor and Leiva-Leon (2016)). However, when dealing with a higher level of disaggregation, e.g. at regional level, the inherent larger degree of heterogeneity in the data may lead to nonlinear relationships between the variables under study. Therefore, to provide robust assessments of the relationship between similarities in regional

\footnotetext{
${ }^{29}$ Clark and van Wincoop (2001) confirm the existence of a border effect on within-country correlations (of some French and German regions) larger than that on cross-country correlations. Acedo-Montoya and de Haan (2011) find that within-country cycles are always more correlated than they are with the euro area cycle, their sample being 53 regions (12 countries). All these previous findings contradict those of Fatas (1997) who suggests than the effect of national borders has been reduced over time for 38 regions ( 4 countries).
}

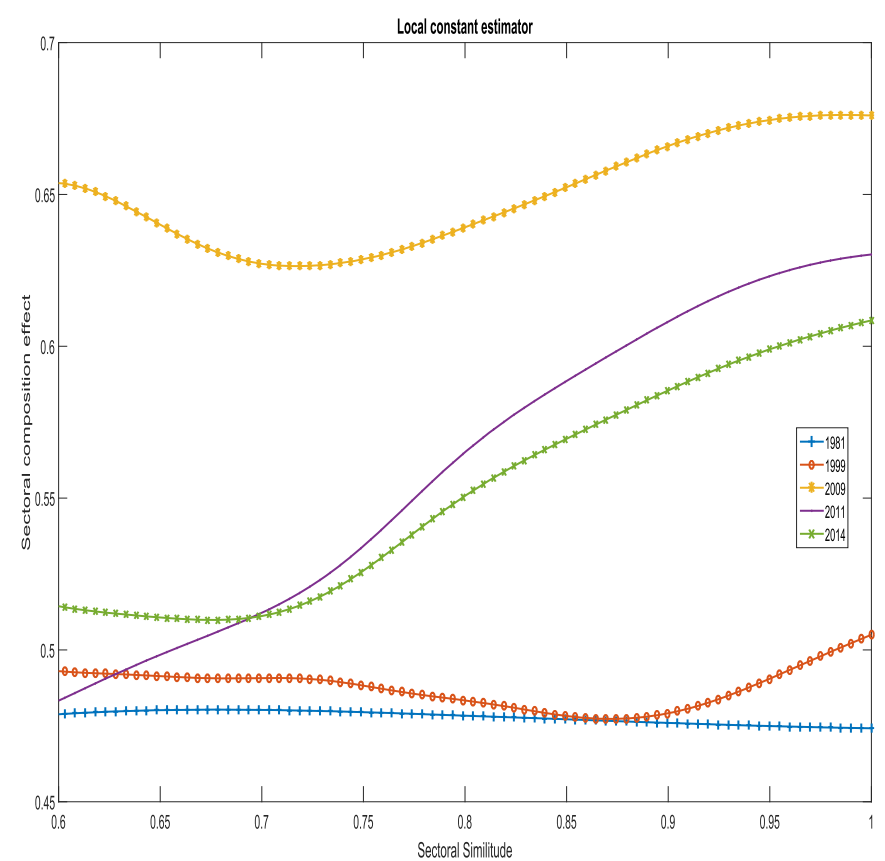

(a) Conditional mean

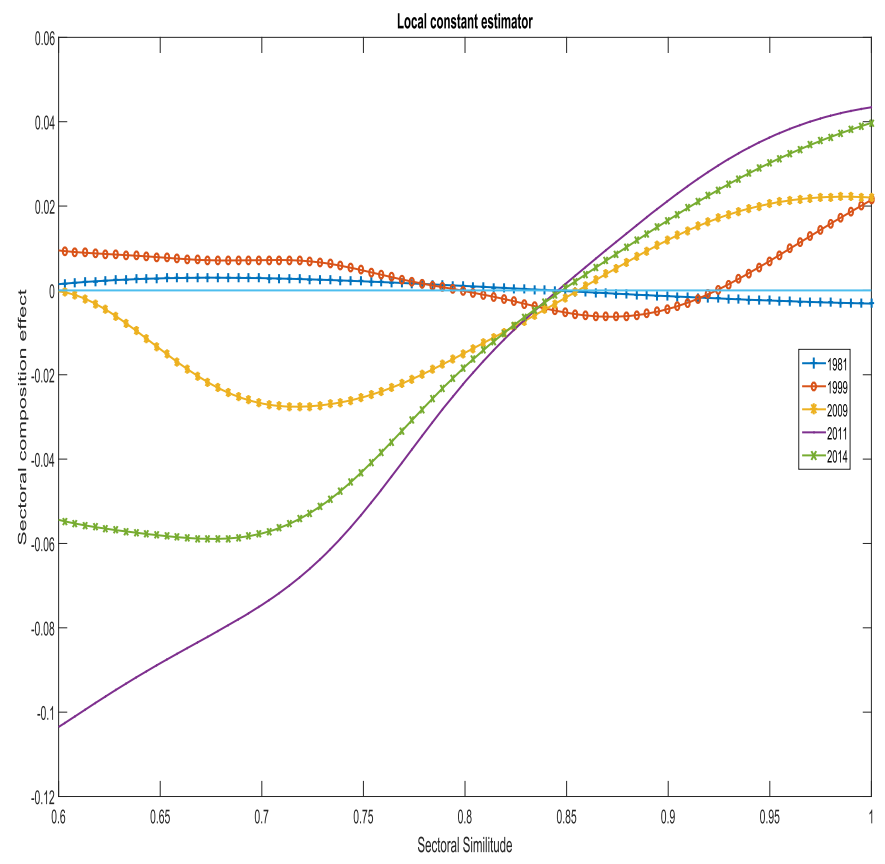

(b) Conditional minus unconditional mean

Fig. 7. Expected synchronisation conditional on sectoral similarity. Note. Chart (a) shows the conditional expectation of synchronisation for selected periods of time, that is, $E_{t}\left(\delta_{t}^{a b} \mid C_{t}^{a b}=c\right)$. Chart (b) shows the conditional minus the unconditional expectation of synchronisation for selected periods of time, that is, $\Delta_{t}^{a b}=E_{t}\left(\delta_{t}^{a b} \mid C_{t}^{a b}=c\right)-E_{t}\left(\delta_{t}^{a b}\right)$.

business cycles, $\delta_{t}^{a b}$, and similarities in regional sectoral composition, $C_{t}^{a b}$, we take advantage of the rich set of information, composed of 22,578 pairwise linkages between the 213 NUTS-2 European regions, and make minimal assumptions by employing nonparametric methods. 

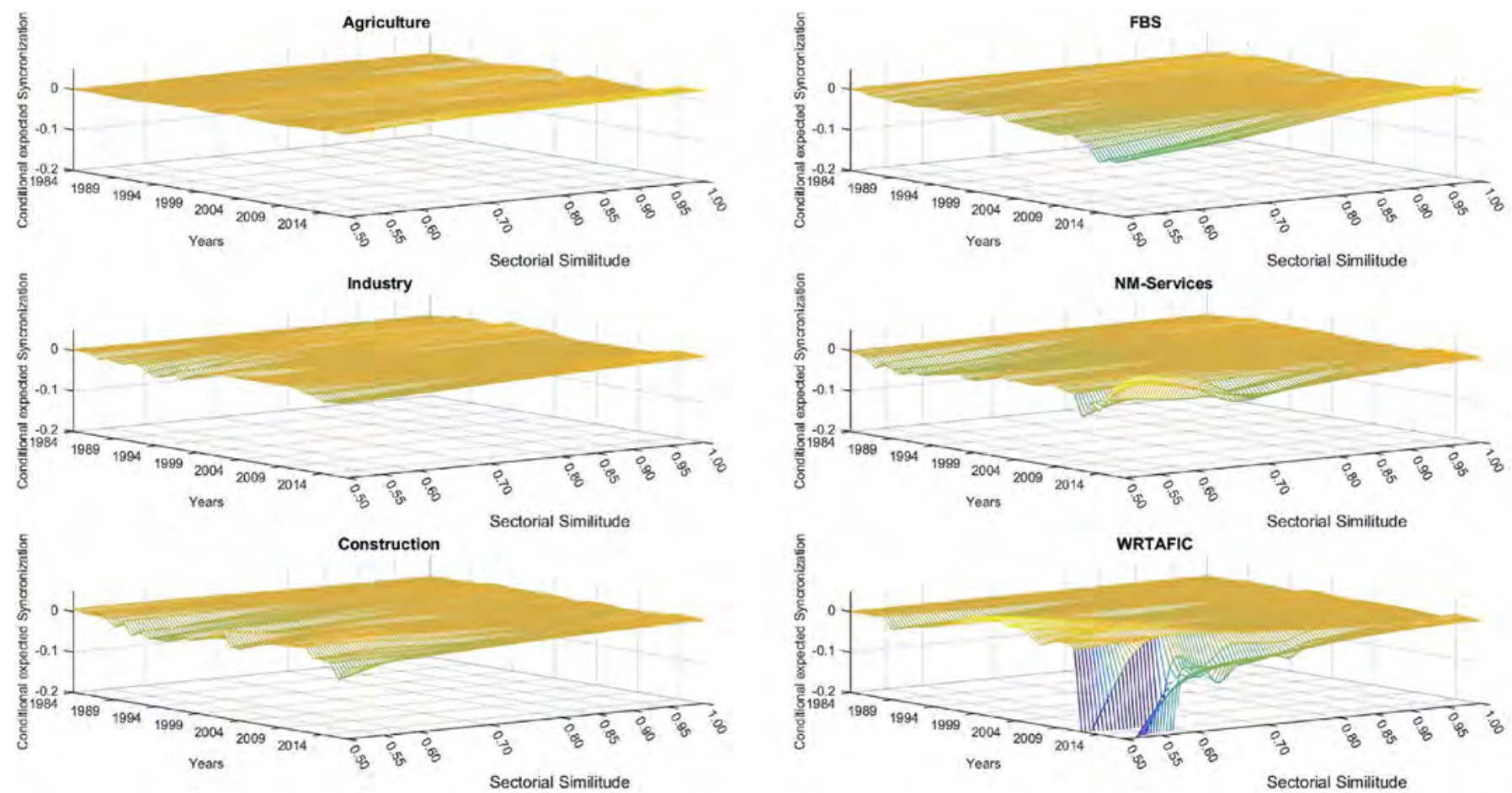

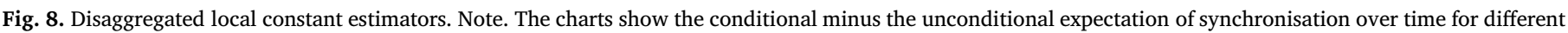
sectors.

Our focus is on assessing the role of similarities in regional sectoral composition in explaining regional business cycle synchronisation patterns and how this role has evolved over time. In doing so, we first compute the average synchronisation over each possible level of sectoral composition similarity, $E_{t}\left(\delta_{t}^{a b} \mid C_{t}^{a b}=c\right)$. Notice that this conditional expectation can be calculated for each period of time, $t=1,2, \ldots, T$. To compute the conditional expectation, we use the Nadaraya-Watson estimator (Local Constant) with a Gaussian kernel. The selection of the optimal bandwidth is based on cross-validation using the Quartic kernel for each year. The results show two main features that are illustrated in Chart (a) of Fig. 7. First, from the early 1980s until the late 2000s, the expected synchronisation remained at moderate levels, around 0.5. However, after 2009, the average synchronisation increased to levels of around 0.7. Second, before the Great Recession, the expected synchronisation remained relatively constant across the different levels of sectoral composition but, after the Great Recession, there has been a clear positive relationship between sectoral similarities and business cycle synchronisation. ${ }^{30}$

Notice that, if $\delta^{a b}$ is independent from $C^{a b}$, then $E\left(\delta^{a b}\right.$ $\left.C^{a b}\right)=E\left(\delta^{a b}\right)$. Therefore, we can measure the role of sectoral similarities in explaining regional synchronisation with the difference between the conditional and the unconditional expectations,

$\Delta_{t}^{a b}(c)=E_{t}\left(\delta_{t}^{a b} \mid C_{t}^{a b}=c\right)-E_{t}\left(\delta_{t}^{a b}\right)$,

where the unconditional expectation is calculated as the simple crosssectional average. Since $\Delta_{t}^{a b}(c)$ can be also computed for $t=1,2, \ldots, T$, we can investigate how the role of sectoral similarities has evolved over time. When $\Delta_{t}^{a b}(c)$ is close to zero, it means that the explanatory power of sectoral similarities is negligible. Conversely, the more different $\Delta_{t}^{a b}(c)$ is from zero, the more informative sectoral similarity is to explain synchronisation patterns. Chart (b) of Fig. 7 plots $\Delta_{t}^{a b}(c)$ from

\footnotetext{
30 Fig. 7 shows the expected synchronisation conditional on the domain of sectoral similarities corresponding to the interval $[0.7,0.9]$ since most of the mass of the distribution is concentrated in that interval, as shown in Fig. 5.
}

selected years, reinforcing the evidence that the propagation of business cycle shocks among European regions has become more dependent on the regional productive structure after the Great Recession. These results are consistent with the findings in Section 4.2.

Moreover, chart (b) of Fig. 7 reveals that the Great Recession triggered a nonlinear relationship between synchronisation and sectoral similarities, as can be seen in $\Delta_{t}^{a b}(c)$ for $t=2009,2011,2014$. This relationship is convex for values of sectoral similarity lower than 0.8 , while it becomes concave for values of sectoral similarity higher than 0.8. ${ }^{31}$ This means that increases in sectoral similarity between regions that already exhibit either very similar $\left(C^{a b}>0.9\right)$ or very different $\left(C^{a b}<0.7\right)$ productive structures are expected to yield small increases in regional business cycle synchronisation. However, increases in sectoral similarity between regions that show moderately similar productive structures $\left(0.7<C^{a b}<0.9\right)$ are expected to yield large increases in regional business cycle synchronisation. These results have important implications for the Cohesion Policy of the European Union whose main objectives are to avoid regional disparities, such as dissimilarities in regional business cycle fluctuations, and to restructure declining industrial areas. ${ }^{32}$

The non-parametric analysis has provided information about the type of relationship between $\delta^{a b}$ and $C^{a b}$, making minimal assumptions, and has allowed us to quantify the sectoral composition effect more accurately than using parametric regression analysis. This framework has also enabled us to identify the nonlinear effect of the sectoral composition hidden in linear panel data analysis or time-dependent regressions. Next, we analyse more deeply the source of this nonlinear effect. We want to identify the sector or sectors that most contribute to this nonlinear effect of sectoral composition on business cycle synchronisation. To do so, we disaggregate the productive structure of the regions

\footnotetext{
31 The figures are around 0.8 but vary depending on the year.

32 For robustness, we have also estimated the conditional expectation using the Local Linear estimator proposed by Stone (1977) instead of the Local Constant method. The results are plotted in Fig. 13 in Appendix D and reach the same conclusions. For a discussion of both methods see Liu (2011).
} 
into the corresponding sectors, as follows,

$C_{t}^{i, a b}=1-\frac{\left|c_{t}^{i, a}-c_{t}^{i, b}\right|}{c_{t}^{i, a}+c_{t}^{i, b}}$,

where $C_{t}^{i, a b}$ is the contribution of sector $i$ to the sectoral composition similarity between regions $a$ and $b$. As in the aggregate case, this index ranges from 0 to 1 , taking the value of 0 , when the shares associated with sector $i$ of regions $a$ and $b$ are radically different, and 1 when the shares of sector $i$ are identical for both regions. Accordingly, we measure the contribution of similarities in each sector to explaining regional synchronisation by computing,

$\Delta_{t}^{i, a b}(c)=E_{t}\left(\delta_{t}^{a b} \mid C_{t}^{i, a b}=c^{i}\right)-E_{t}\left(\delta_{t}^{a b}\right)$,

for $i=$ Agriculture, Industry, Construction, WRTAFIC, FBS, NMservices. In order to provide a full characterization of the contribution of each sector for each period of time, we use 3-D plots. Fig. 8 shows $\Delta_{t}^{i, a b}(c)$ estimated with the Local Constant method for $t=1,2, \ldots, T$. The figure indicates that the indexes $\Delta_{t}^{i, a b}(c)$ associated with the sectors of Agriculture, Industry and Construction have an almost flat pattern around zero, while, the indexes associated with the sectors of Wholesale, retail, transport, accommodation and food services, information and communication (WRTAFIC), Financial and business services (FBS), and Non-market services exhibit a nonlinear pattern, especially after 2009. ${ }^{33}$ Therefore, the evolution of synchronisation among European regions after the Great Recession could be due to the convergence of regional economic landscapes towards similar service-oriented economies.

This finding is related to the process of structural transformation, which is, in turn, linked to the reallocation of economic activity across different sectors. In particular, previous studies have shown that, in recent years, increases in per capita GDP have been associated with decreases in both the employment and nominal value added shares in the agricultural sector, and increases in both the employment and the nominal value added shares in the service sector. The manufacturing sector has behaved differently from the other two in that its employment and nominal value added shares follow a hump shape, that is, they are increasing for lower levels of development and decreasing for higher levels of development of a given country. ${ }^{34}$ Consequently, as the services sector has gained importance in the developments of real activity, it would make sense that it is the main source of business cycle synchronisation at the regional level. The progressive advance of services in terms of employment and production, the liberalization of international trade in services and the high strategic value of certain tertiary activities (telecommunications, business services, transports, etc.) stress the great importance of the service sector in regional and national economic growth.

\section{Conclusions}

In this paper, we analyse changes in the synchronisation of European regional business cycles and investigate the role of sectoral composition and other explanatory factors in explaining these changes. We also identify the regions most interrelated with the rest of Europe. Our sample has a wider and more disaggregated geographical and temporal coverage than previous research. Due to the lack of high frequency data at the regional level, we propose a new method to measure time-varying synchronisation in small samples that combines regime-switching models and dynamic model averaging. The reliability of the proposed method is validated with Monte Carlo experiments.

The main results show that Great Recession was responsible for twice as much synchronisation as the European Union integration process caused in several decades. Moreover, we show that this increase in synchronisation was not temporary because regional interdependence has remained at much higher levels after the end of the Great Recession. Regarding interconnectedness, we also show that Ile de France acts as the most central region in the propagation of business cycle shocks in Europe, followed by Inner London and Lombardia.

When assessing some factors of synchronisation, we find that the similarity of regional sectoral composition is the main factor that explains regional synchronisation in Europe, especially since the Great Recession. Moreover, the Great Recession triggered a nonlinear relationship between synchronisation and sectoral similarities, which reinforces the idea that the propagation of business cycle shocks among European regions has become more dependent on the regional productive structure after the Great Recession. Indeed, similarities in servicesrelated industries across regions are primarily responsible for this nonlinear relationship.

Sectoral composition has significant implications for aggregate economic fluctuations because the observed increase of sectoral homogeneity among regions, as time progresses, amplifies the effects of sectoral shocks at the aggregate level. The robustness of our results strengthens the link between the regional productive structure and the synchronisation of business cycle shocks. Our findings provide crucial information for policymakers in the implementation of the Cohesion Policy as we not only offer a comprehensive framework of regional dynamics in the last three decades but also determine which economic regions are the most sensitive to policy changes or shocks.

\section{Appendix A}

Priors

For the mean and variance parameters in vector $\theta$, the independent Normal-Wishart prior distribution is used:

$p\left(\mu, \Sigma^{-1}\right)=p(\mu) p\left(\Sigma^{-1}\right)$,

where

$$
\begin{aligned}
\mu & \sim N\left(\underline{\mu}, \underline{V}_{\mu}\right) \\
\Sigma^{-1} & \sim W\left(\underline{S}^{-1}, \underline{\mathrm{v}}\right),
\end{aligned}
$$

and the associated hyperparameters are given by $\underline{\mu}=(-1,2-1,2)^{\prime}, \underline{V}_{\mu}=I, \underline{S}^{-1}=I, \underline{v}=0 .^{35}$

For the transition probabilities $p_{a, 00}, p_{a, 11}$, of $S_{a, t}, p_{b, 00}, p_{b, 11}$, of $S_{b, t}$, and $p_{00}, p_{11}$, of $S_{t}$, Beta distributions are used as conjugate priors:

\footnotetext{
${ }^{33}$ We also perform the same exercise using the Local Linear estimator, but the

results remain unchanged, as can be seen in Fig. 13 in Appendix D.

${ }^{34}$ See Herrendorf et al. (2014) for a detailed review of the literature on this structural transformation.

${ }^{35}$ For the empirical application, due to the substantial heterogeneity in the magnitude of growth rates at the regional level, we use $\mu=\left(\mu_{a^{-}}, \mu_{a^{+}}, \mu_{b^{-}}, \mu_{b^{+}}\right)$as hyperparameter priors for the means, where $\mu_{t^{-}}$and $\mu_{l^{+}}$are the means of positive and negative values of $y_{l, t}$, respectively, for $l=a, b$.
} 
$p_{k, 00} \sim B e\left(u_{k, 11}, u_{k, 10}\right), p_{k, 11} \sim B e\left(u_{k, 00}, u_{k, 01}\right)$, for $k=a, b$

$p_{00} \sim \operatorname{Be}\left(u_{11}, u_{10}\right), p_{11} \sim \operatorname{Be}\left(u_{00}, u_{01}\right)$,

where the hyperparameters are given by $u_{a, 01}=u_{b, 01}=u_{01}=2, u_{a, 00}=u_{b, 00}=u_{00}=8, u_{a, 10}=u_{b, 10}=u_{10}=1$ and $u_{a, 11}=u_{b, 11}=u_{11}=9$.

To be able to generate draws of the forgetting factor, we linearize the equation associated with the dynamics of $\widetilde{\delta}_{T}$ and treat it as a linear regression, for which we use a Normal-Gamma prior distribution given by:

$\alpha \sim N\left(\underline{\alpha}, \underline{\sigma}_{\alpha}\right)$

$\sigma_{\delta}^{-1} \sim G\left(\underline{s}^{-1}, \underline{v}\right)$,

with hyperparameters associated with the Normal distribution, $\underline{\alpha}=0.9, \underline{\sigma}_{\alpha}=0.1$, and with the Gamma distribution, $s^{-1}=0, \underline{v}=0$.

Gibss sampler

$\operatorname{Draw} \widetilde{S}_{a, T}, \widetilde{S}_{b, T}, \widetilde{S}_{T}, \widetilde{\delta}_{T}$

In order to make inferences about the variables $\widetilde{S}_{a, T}, \widetilde{S}_{b, T}, \widetilde{S}_{T}$ and $\widetilde{\delta}_{T}$, we follow the line of Kim and Nelson (1999) and compute draws from the conditional distributions:

$g\left(\widetilde{S}_{k, T} \mid \theta, \tilde{y}_{T}\right)=g\left(S_{k, T} \mid \tilde{y}_{T}\right) \prod_{t=1}^{T} g\left(S_{k, t} \mid S_{k, t+1}, \tilde{y}_{t}\right)$, for $k=a, b$

$g\left(\widetilde{S}_{T} \mid \theta, \tilde{y}_{T}\right)=g\left(S_{T} \mid \tilde{y}_{T}\right) \prod_{t=1}^{T} g\left(S_{t} \mid S_{t+1}, \tilde{y}_{t}\right)$.

In order to obtain the two terms on the right-hand side of Equations (28) and (29), the following two steps can be employed:

Step 1: Conditional on the parameters $\theta$, we run the filtering algorithm proposed in Section 2.2, get a draw of $\widetilde{\delta}_{T}$, and compute the terms $g\left(\widetilde{S}_{k, t} \mid \widetilde{y}_{t}\right)$ for $k=a, b$, and $g\left(\widetilde{S}_{t} \mid \widetilde{y}_{t}\right)$ for $t=1,2, \ldots, T$, save them and take the elements for which $t=T$.

Step 2: The product in the second term of the right-hand side of Equations (28) and (29) can be obtained for $t=T-1, T-2, \ldots, 1$ by following the result:

$$
\begin{aligned}
g\left(S_{t} \mid \tilde{y}_{t}, S_{t+1}\right) & =\frac{g\left(S_{t}, S_{t+1} \mid \tilde{y}_{t}\right)}{g\left(S_{t+1} \mid \tilde{y}_{t}\right)} \\
& \propto g\left(S_{t+1} \mid S_{t}\right) g\left(S_{t} \mid \tilde{y}_{t}\right),
\end{aligned}
$$

where $g\left(S_{t+1} \mid S_{t}\right)$ corresponds to the transition probabilities of $S_{t}$ and $g\left(S_{t} \mid \tilde{y}_{t}\right)$ saved in Step 1 .

Then, we compute

$\operatorname{Pr}\left[S_{t}=1 \mid S_{t+1}, \tilde{y}_{t}\right]=\frac{g\left(S_{t+1} \mid S_{t}=1\right) g\left(S_{t}=1 \mid \tilde{y}_{t}\right)}{\sum_{j=0}^{1} g\left(S_{t+1} \mid S_{t}=j\right) g\left(S_{t}=j \mid \tilde{y}_{t}\right)}$

and generate a random number from $U[0,1]$. If that number is less than or equal to $\operatorname{Pr}\left[S_{t}=1 \mid S_{t+1}, \tilde{y}_{t}\right]$, then $S_{t}=1$, otherwise $S_{t}=0$. The same procedure applies for $S_{a, t}$, and $S_{b, t}$.

Draw $p_{a, 00}, p_{a, 11}, p_{b, 00}, p_{b, 11}, p_{00}, p_{11}$

Conditional on $\widetilde{S}_{k, T}$ for $k=a, b$ and $\widetilde{S}_{T}$, the transition probabilities are independent from the data set and the model's parameters. Hence, focusing on the case of $\widetilde{S}_{T}$, the likelihood function of $p_{00}, p_{11}$ is given by

$L\left(p_{00}, p_{11} \mid \widetilde{S}_{T}\right)=p_{00}^{n_{00}}\left(1-p_{00}^{n_{01}}\right) p_{11}^{n_{11}}\left(1-p_{11}^{n_{10}}\right)$,

where $n_{i j}$ refers to the transitions from state $i$ to $j$, accounted for in $\widetilde{S}_{T}$.

Combining the prior distribution with the likelihood, the posterior distribution is given by

$p\left(p_{00}, p_{11} \mid \widetilde{S}_{T}\right) \propto p_{00}^{u_{00}+n_{00}-1}\left(1-p_{00}\right)^{u_{01}+n_{01}-1} p_{11}^{u_{11}+n_{11}-1}\left(1-p_{11}\right)^{u_{10}+n_{10}-1}$,

which indicates that draws of the transition probabilities will be taken from

$p_{00}\left|\widetilde{S}_{T} \sim B e\left(u_{00}+n_{00}, u_{01}+n_{01}\right), p_{11}\right| \widetilde{S}_{T} \sim B e\left(u_{11}+n_{11}, u_{10}+n_{10}\right)$.

The same procedure applies for the cases of $\widetilde{S}_{k, T}$ for $k=a, b$.

Draw $\mu_{0, a}, \mu_{1, a}, \mu_{0, b}, \mu_{1, b}$

The bivariate Markov-switching model can be expressed compactly as

$\left[\begin{array}{l}y_{a, t} \\ y_{b, t}\end{array}\right]=\left[\begin{array}{cccc}1 & S_{a, t} & 0 & 0 \\ 0 & 0 & 1 & S_{b, t}\end{array}\right]\left[\begin{array}{l}\mu_{a, 0} \\ \mu_{a, 1} \\ \mu_{b, 0} \\ \mu_{b, 1}\end{array}\right]+\left[\begin{array}{l}e_{a, t} \\ e_{b, t}\end{array}\right], \quad\left[\begin{array}{l}e_{a, t} \\ e_{b, t}\end{array}\right] \sim N\left(\left[\begin{array}{l}0 \\ 0\end{array}\right],\left[\begin{array}{cc}\sigma_{a}^{2} & \sigma_{a b} \\ \sigma_{a b} & \sigma_{b}^{2}\end{array}\right]\right)$

$y_{t}=\bar{S}_{t} \mu+\xi_{t}, \xi_{t} \sim N(\mathbf{0}, \Sigma)$,

stacking it as 
$y=\left[\begin{array}{c}y_{1} \\ y_{2} \\ \vdots \\ y_{T}\end{array}\right], \bar{S}=\left[\begin{array}{c}\bar{S}_{1} \\ \bar{S}_{2} \\ \vdots \\ \bar{S}_{T}\end{array}\right]$, and $\xi=\left[\begin{array}{c}\xi_{1} \\ \xi_{2} \\ \vdots \\ \xi_{T}\end{array}\right]$.

The model in Equation (35) remains written as a normal linear regression model with an error covariance matrix of a peculiar form:

$y=S \mu+\xi, \xi \sim N(\mathbf{0}, I \otimes \Sigma)$

Conditional on the covariance matrix parameters, state variables and the data, by using the corresponding likelihood function, the posterior distribution $p\left(\mu \mid \widetilde{S}_{a, T}, \widetilde{S}_{b, T}, \widetilde{S}_{T}, \widetilde{\delta}_{T}, \Sigma^{-1}, \widetilde{y}_{T}\right)$ takes the form

$\mu \mid \widetilde{S}_{a, T}, \widetilde{S}_{b, T}, \widetilde{S}_{T}, \widetilde{\delta}_{T}, \Sigma^{-1}, \widetilde{y}_{T} \sim N\left(\bar{\mu}, \bar{V}_{\mu}\right)$

where

$\begin{aligned} \bar{V}_{\mu} & =\left(\underline{V}_{\mu}^{-1}+\sum_{t=1}^{T} \bar{S}_{t}^{\prime} \Sigma^{-1} \bar{S}_{t}\right)^{-1} \\ \bar{\mu} & =\bar{V}_{\mu}\left(\underline{V}_{\mu}^{-1} \underline{\mu}+\sum_{t=1}^{T} \bar{S}_{t}^{\prime} \Sigma^{-1} y_{t}\right) .\end{aligned}$

After drawing $\mu=\left(\mu_{a, 0}, \mu_{a, 1}, \mu_{b, 0}, \mu_{b, 1}\right)^{\prime}$ from the above multivariate distribution, if the generated value of $\mu_{a, 1}$ or $\mu_{b, 1}$ is less than or equal to 0 , that draw is discarded; otherwise, it is saved, in order to ensure that $\mu_{a, 1}>0$ and $\mu_{b, 1}>0$.

Draw $\sigma_{a}^{2}, \sigma_{b}^{2}, \sigma_{a b}$

Conditional on the mean parameters, state variables and the data, by using the corresponding likelihood function, the posterior distribution $p\left(\Sigma^{-1} \mid \widetilde{S}_{a, T}, \widetilde{S}_{b, T}, \widetilde{S}_{T}, \widetilde{\delta}_{T}, \mu, \widetilde{y}_{T}\right)$ takes the form

$\Sigma^{-1} \mid \widetilde{S}_{a, T}, \widetilde{S}_{b, T}, \widetilde{S}_{T}, \widetilde{\delta}_{T}, \mu, \tilde{y}_{T} \sim W\left(\bar{S}^{-1}, \bar{v}\right)$,

where

$\overline{\mathrm{v}}=T+\underline{\mathrm{v}}$

$\bar{S}=\underline{S}+\sum_{t=1}^{T}\left(y_{t}-\bar{S}_{t} \mu\right)\left(y_{t}-\bar{S}_{t} \mu\right)^{\prime}$.

After $\Sigma^{-1}$ is generated, the elements in $\Sigma$ are recovered.

Draw $\alpha$

In our context of finite samples, small variations in $\alpha$ may end up significantly affecting the dynamics of $\delta_{t}$. To avoid this drawback, instead of simply imposing a given forgetting factor $\alpha$, we use the Bayesian procedure to estimate the other elements of the model and generate draws to simulate the posterior distribution of $\alpha$. We linearize the predicted model probability $\pi_{t+1 \mid t, D}=\delta_{t+1 \mid t}$ in equation (13) to relate it to the updated model probability $\pi_{t \mid t, D}=\delta_{t \mid t}$ in equation (9) with the following linear regression:

$\ln \left(\delta_{t+1 \mid t}\right)=\alpha \ln \left(\delta_{t \mid t}\right)+\varepsilon_{t}$

where $\varepsilon_{t}$ is assumed to be normally distributed with variance $\sigma_{\delta}$. Conditional on the variance $\sigma_{\delta}$, using the corresponding likelihood function, the posterior distribution $p\left(\alpha \mid \sigma_{\delta}, \widetilde{\delta}_{T}\right)$ takes the form

$\alpha \mid \sigma_{\delta}, \widetilde{\delta}_{T} \sim N\left(\bar{\alpha}, \bar{\sigma}_{\alpha}\right)$,

where

$\begin{aligned} \bar{\sigma}_{\alpha} & =\left(\underline{\sigma}_{\alpha}^{-1}+\sigma_{\delta}^{-1} \sum_{t=1}^{T} \ln \left(\delta_{t \mid t}\right)^{2}\right)^{-1} \\ \bar{\alpha} & =\bar{\sigma}_{\alpha}\left(\underline{\sigma}_{\alpha}^{-1} \underline{\alpha}+\sigma_{\delta}^{-1} \sum_{t=1}^{T} \ln \left(\delta_{t \mid t}\right) \ln \left(\delta_{t+1 \mid t}\right)\right) .\end{aligned}$

After obtaining a draw of $\alpha$ from the above posterior distribution, if $0<\alpha<1$, it is saved; otherwise the draw is discarded to ensure the interpretation of a forgetting factor.

Draw $\sigma_{\delta}$

Conditional on the parameter $\alpha$, and using the corresponding likelihood function, the posterior distribution $p\left(\sigma_{\delta}^{-1} \mid \alpha, \widetilde{\delta}_{T}\right)$ takes the form

$\sigma_{\delta}^{-1} \mid \alpha, \widetilde{\delta}_{T} \sim G\left(\bar{s}^{-1}, \bar{v}\right)$,

where

$\bar{v}=T+\underline{v}$ 
$\bar{s}=\underline{s}+\sum_{t=1}^{T}\left(\ln \left(\delta_{t+1 \mid t}\right)-\alpha \ln \left(\delta_{t \mid t}\right)\right)^{2}$.

After $\sigma_{\delta}^{-1}$ is generated, the variance $\sigma_{\delta}$ is recovered.

\section{Appendix B}

The United Kingdom voted to leave the European Union following the June 2016 referendum. With the exit of the UK from the EU, an event commonly known as "Brexit", global economic and policy uncertainty has significantly increased since negative economic consequences, not only for the UK but also for the economies that remain in the EU, are expected. Considering this scenario, it is interesting to analyse the relationship between the UK and the European regions with the most recent available data.

The econometric framework proposed in this paper can be used to characterize these economic relationships. To this end, we consider the following three steps. First, we aggregate the regions of the UK. Second, we estimate its time-varying business cycle interdependence with each of the remaining regions of Europe. Finally, we select the most updated set of estimated synchronisation measures, corresponding to the year 2014, and use a heatmap to visualize this information. Fig. 9 shows the strength of the connections between the UK and the European regions. We find that, in 2014, the regions that exhibited the strongest connection with the UK were the regions located in Finland, Sweden, West Germany, Northern Italy, Austria and some regions in the middle of France.

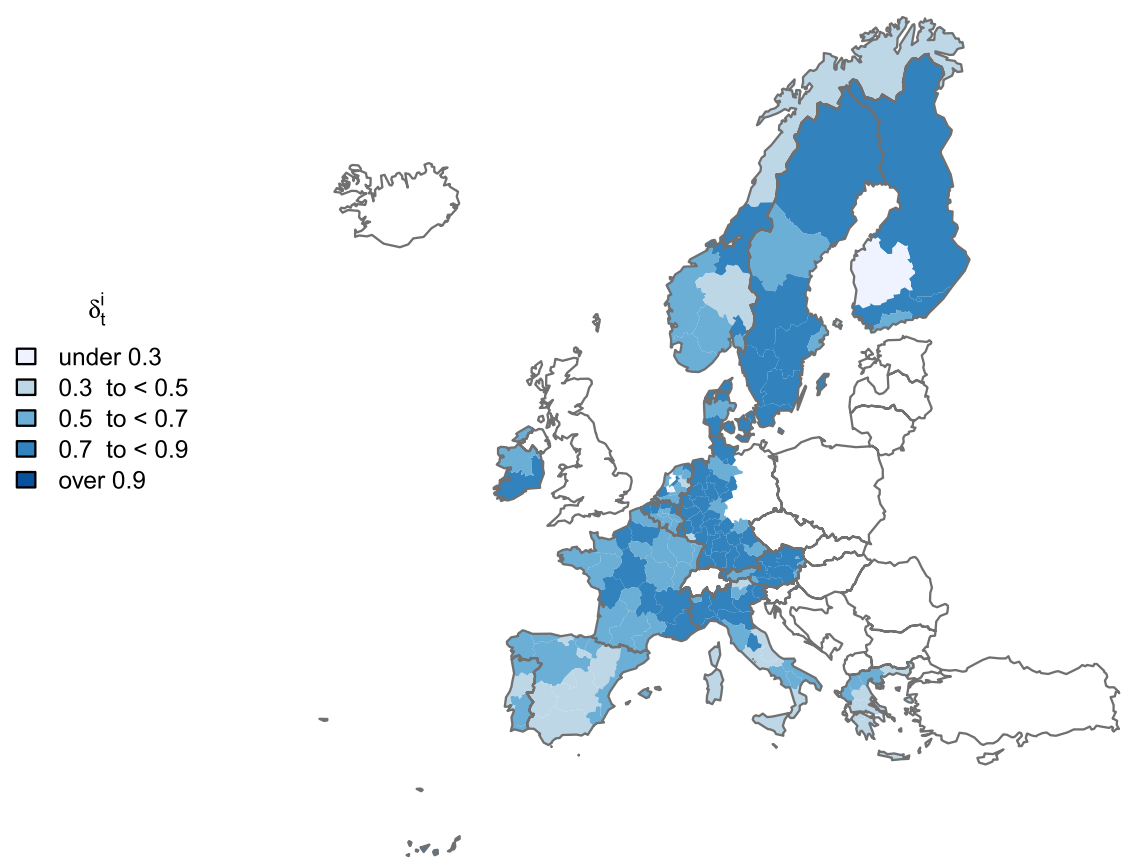

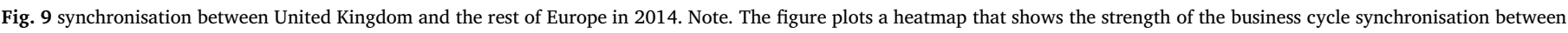
the UK (as a whole) and the European regions. The darker (lighter) each region is, the higher (lower) its synchronisation with the UK is.

\section{Appendix C}

To get a better understanding about the space dimension of European regional synchronisation, we measure the degree of cohesion associated with each country. This exercise allows us to identify the countries that have contributed the most to the increasing European synchronisation pattern. The time-varying cohesion of a given country $\kappa$ at time $t$ can be interpreted as a measure of country-specific internal synchronisation, and it is defined as follows.

$c_{t}^{\kappa}=\frac{\sum_{a \neq b \in \kappa} \omega_{a, t} \omega_{b, t} \delta_{t}^{a b}}{\sum_{a \neq b \in \kappa} \omega_{a, t} \omega_{b, t}}$.

Chart A of Fig. 10 shows the cohesion measures for central European countries (DE, NL, AT, BE), which experienced a progressive increase from 1981 until 2008 and a hike at the beginning of the Great Recession. Chart B shows the cohesion associated with Mediterranean countries (PT, ES, FR, IT, GR), showing a relatively similar pattern to the cohesion of Central countries. Most of these countries are in the eurozone, which would explain this upward trend. Amongst them, Portugal shows the lowest degree of internal synchronisation during the whole sample, while Spain stands out for its high cohesion during most of the period considered. Notice that, after the Great Recession, the cohesion indexes of both central European and Mediterranean countries remained at high levels, showing that the latest global recession had long-lasting consequences for these European regions in terms of business cycle synchronisation. Chart C plots the cohesion for the British Isles (UK, IE), which exhibit a rather different pattern. These countries underwent a significant decline in internal synchronisation during the 1990s and 2000s. At the beginning of the Great Recession, the cohesion in both economies significantly increased. Finally, Chart D plots the cohesion measures of Nordic countries (FI, DK, NO, SE), showing a pattern relatively similarly to that of the British Isles. Nordic countries underwent a decreasing synchronisation from the mid 1990s until 2007 -which was especially sharp for Norway after 1997 and Denmark after 2001. Finland is an exception as it has an overall increasing trend in internal synchronisation throughout the sample, though with a very volatile profile. This may be related to the fact that Finland is the 
only Nordic country that is included in the eurozone. These results suggest that the pattern of increasing synchronisation in Europe can be mainly attributed to the Central and Mediterranean countries, since the British Isles and Nordic countries have a different behaviour.

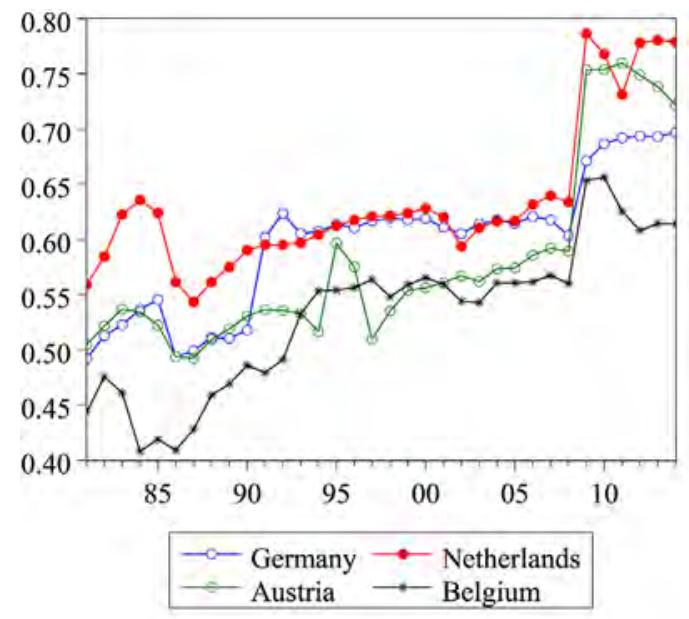

(a)

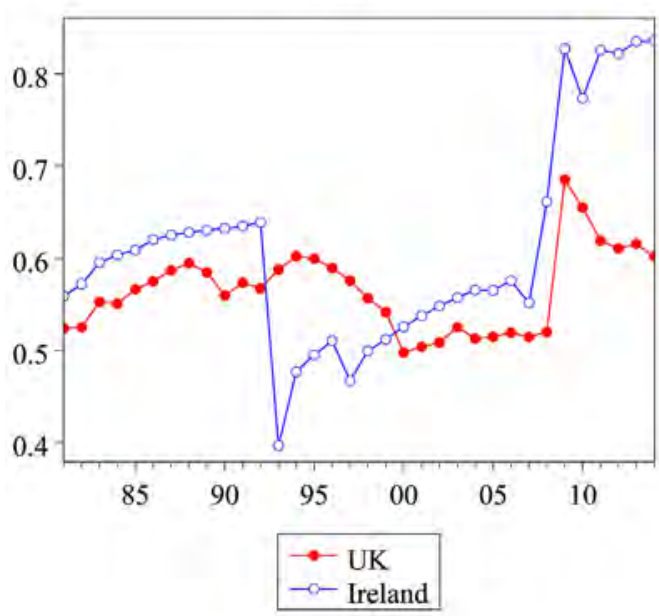

(c)

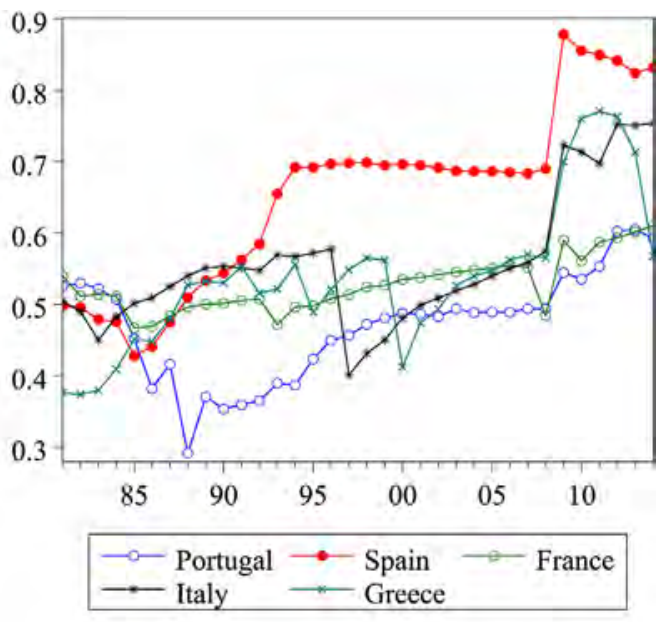

(b)

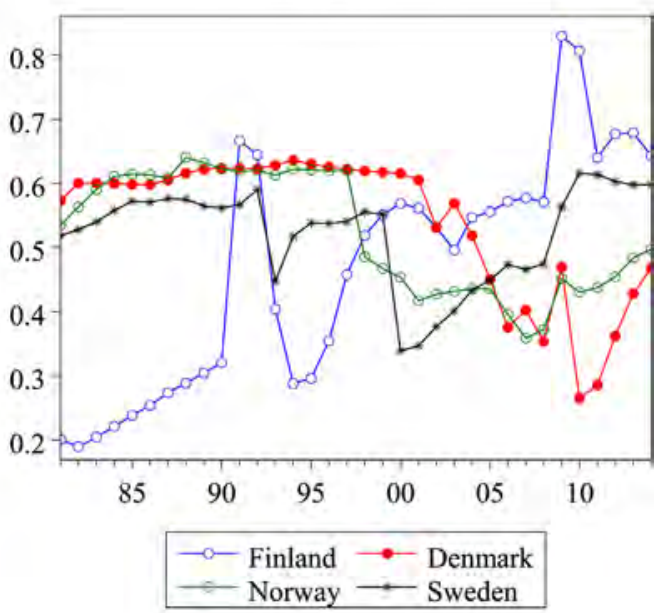

(d)

Fig. 10 Time-varying country-specific cohesion. Note. The figure plots the time-varying cohesion measures for each European country grouped in charts based on their geographic proximity. 


\section{Appendix D}

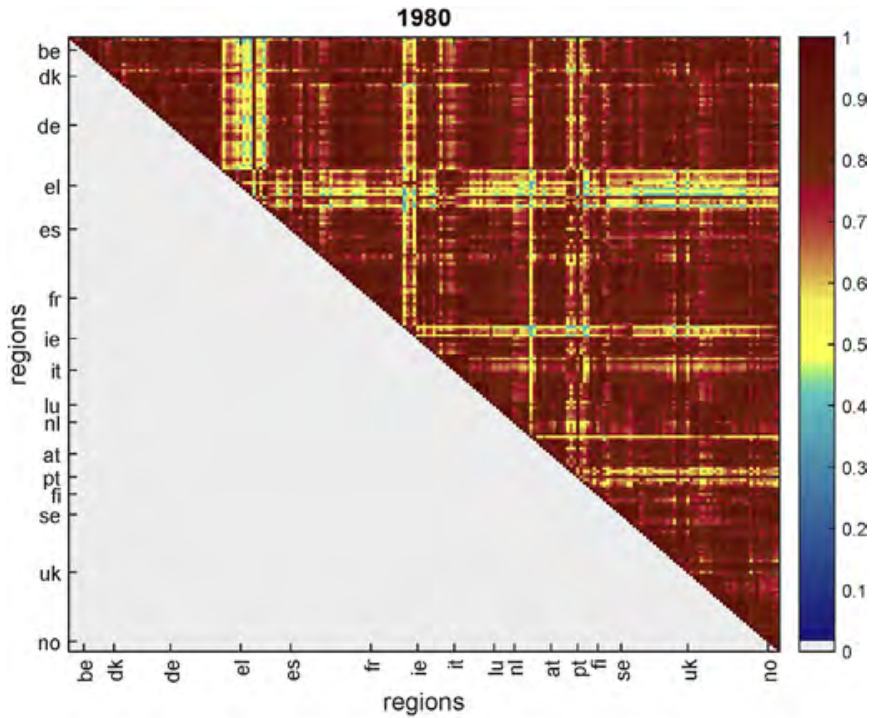

(a) Beginning of the sample: 1980

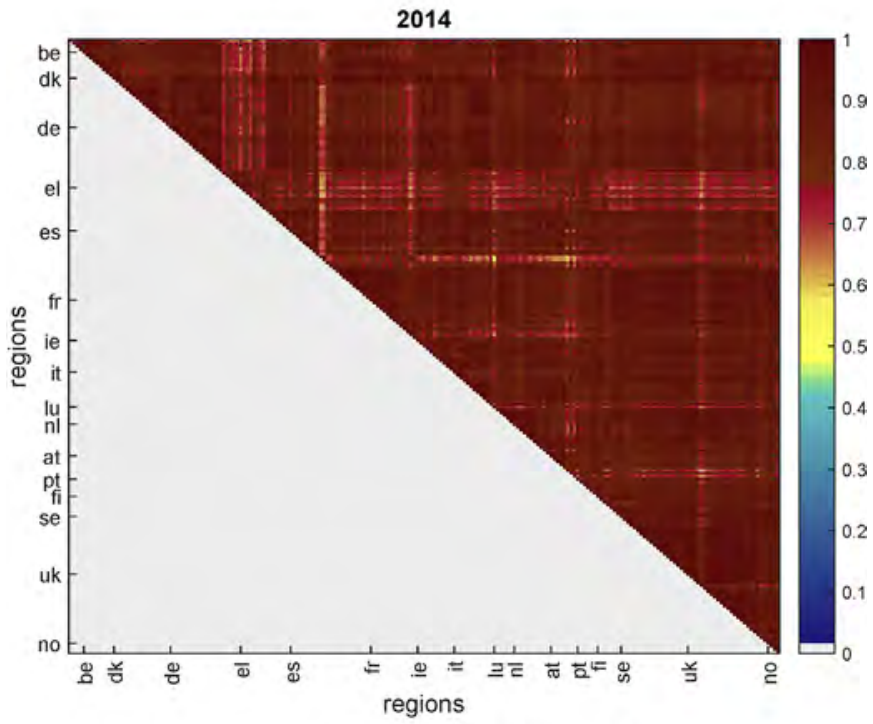

(b) End of the sample: 2014

Fig. 11 Map of sectoral similarity across regions. Note. The heatmaps plot the patterns of sectoral composition similarities between European regions at the beginning and at the end the sample. Sectoral composition similarity is computed as in Imbs (2004). 


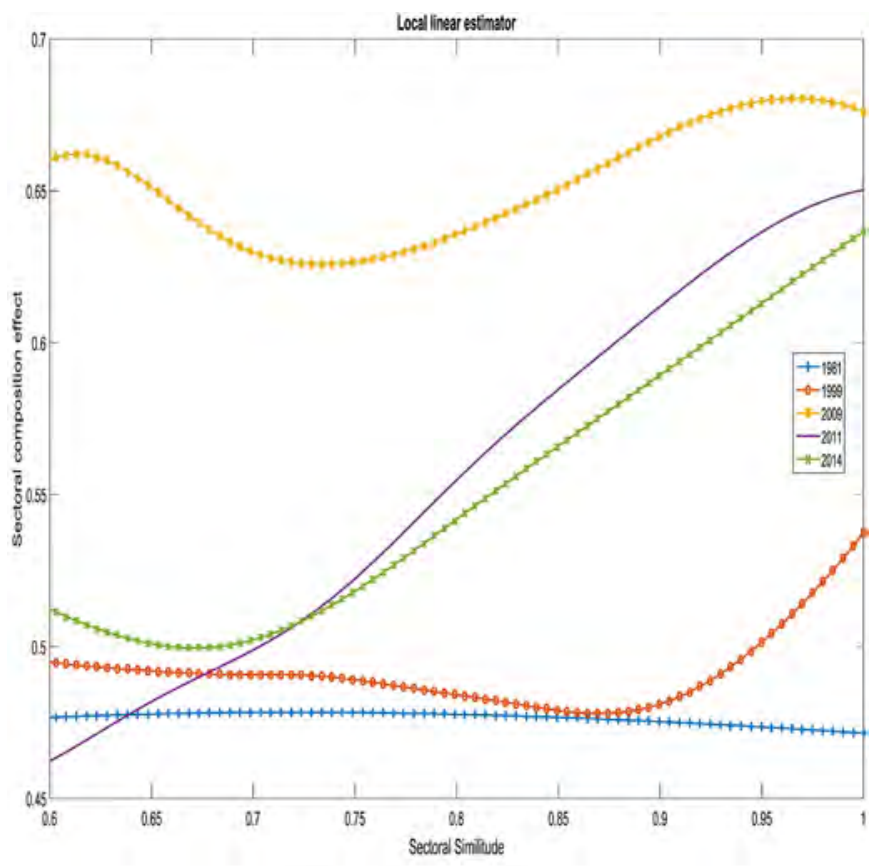

(a) Conditional mean

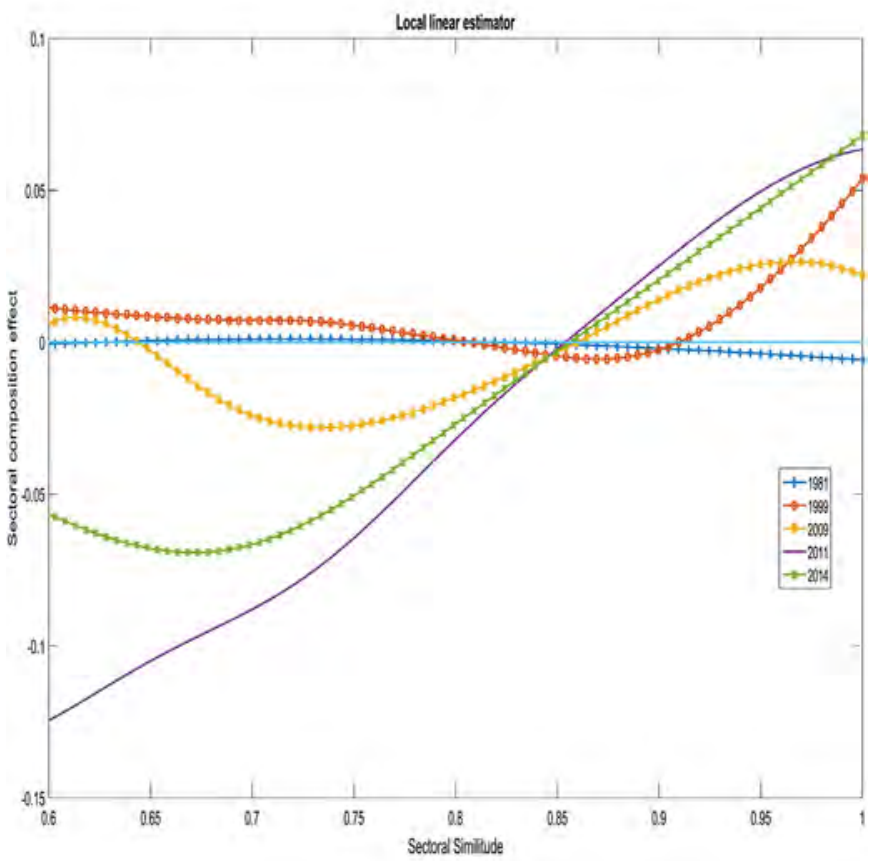

(b) Conditional minus unconditional mean

Fig. 12 Expected synchronisation conditional on sectoral similarity. Note. Chart (a) shows the conditional expectation of synchronisation for selected periods of time, that is, $E_{t}\left(\delta_{t}^{a b} \mid C_{t}^{a b}=c\right)$. Chart (b) shows the conditional minus the unconditional expectation of synchronisation for selected periods of time, that is, $\Delta_{t}^{a b}=E_{t}\left(\delta_{t}^{a b} \mid C_{t}^{a b}=c\right)-E_{t}\left(\delta_{t}^{a b}\right)$. 

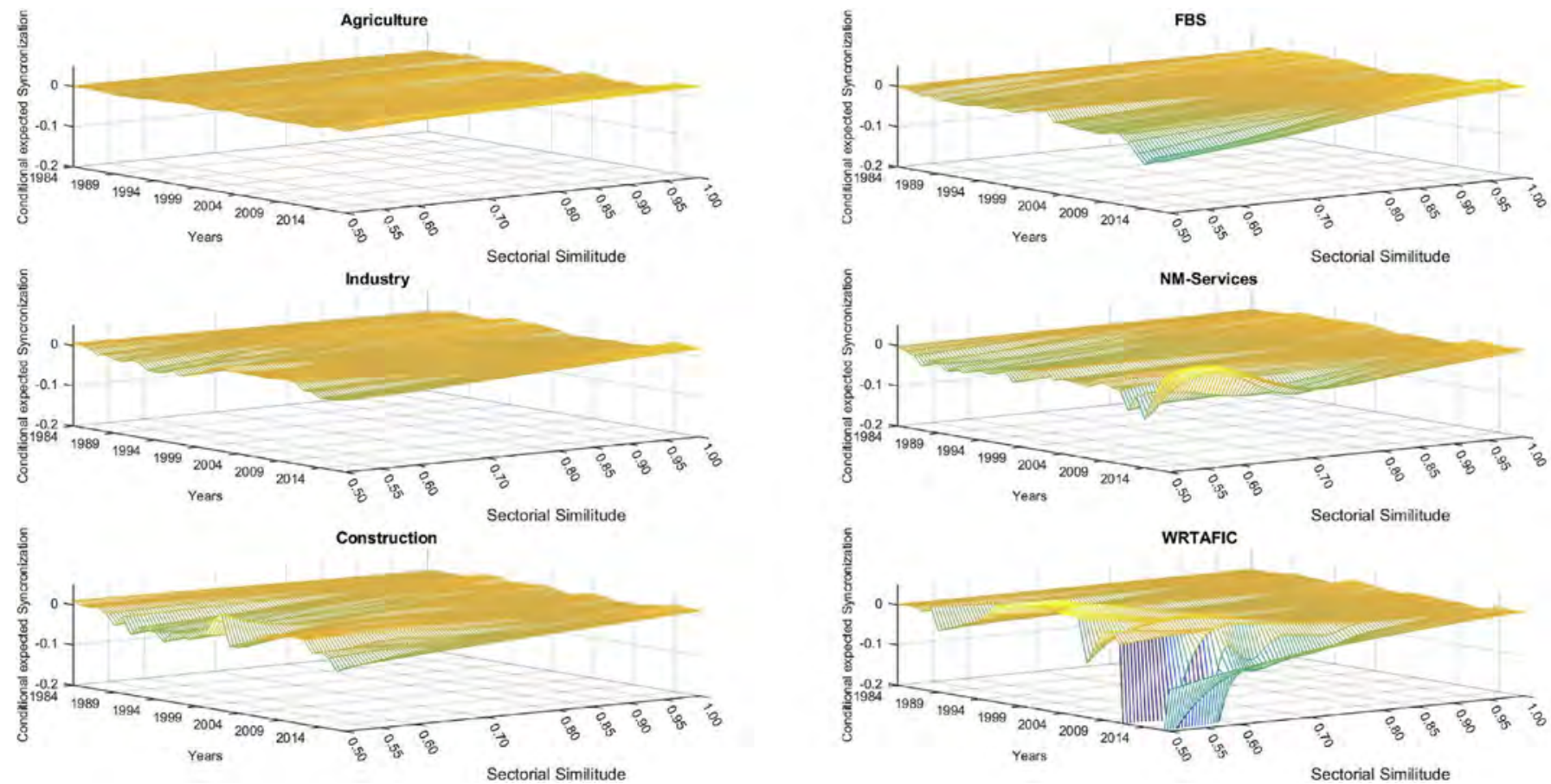

Fig. 13 Disaggregated local linear estimators. Note. The charts show the conditional minus the unconditional expectation of synchronisation over time for different sectors.

\section{Appendix E. Supplementary data}

Supplementary data to this article can be found online at https://doi.org/10.1016/j.econmod.2018.11.009.

\section{References}

Acedo-Montoya, L.A., de Haan, J., 2011. Regional business cycle synchronization in europe? Int. Econ. Econ. Pol. 5 (1-2), 123-137.

Allegret, J.-P., Essaadi, E., 2008. Business cycles synchronization in East Asian economy: evidences from time-varying coherence study. Econ. Modell. 28, 351-365.

Bandres, E., Gadea-Rivas, M.D., Gomez-Loscos, A., 2017. Regional Business Cycles across Europe. Occasional Paper no. 1702. Bank of Spain.

Barrios, S., De Lucio, J.J., 2003. Economic integration and regional business cycles: evidence from the Iberian regions. Oxf. Bull. Econ. Stat. 65, 497-515.

Barrios, S., Brulhart, M., Elliott, R.J.R., Sensier, M., 2003. A tale of two cycles: Co-fluctuations between UK regions and the euro zone. Manch. Sch. 71 (3), 265-292.

Bailey, N., Holly, S., Pesaran, M.H., 2016. A two-stage approach to spatio-temporal analysis with strong and weak cross-sectional dependence. J. Appl. Econom. 31 (1), 249-280.

Belke, A., Heine, J., 2006. Specialization patterns and the synchronicity of regional employment cycles in Europe. Int. Econ. Econ. Pol. 3, 91-104.

Bengoechea, P., Camacho, M., Perez-Quiros, G., 2006. A useful tool for forecasting the Euro-area business cycle phases. Int. J. Forecast. 22, 735-749.

Braudel, F., 1979. Civilization matérielle, économie et capitalisme. XV-XVIII siecle. Tome 2.- Les jeux de l'Echange. Librairie Armand Colin, Paris.

Camacho, M., Leiva-Leon, D., 2017. The propagation of industrial business cycles. Macroecon. Dyn. 37 (12), 2913-2927.

Camacho, M., Perez-Quiros, G., Saiz, L., 2006. Are business cycles close enough to be just one? J. Econ. Dynam. Contr. 30, 1687-1706.

Canova, F., Ciccarelli, M., Ortega, E., 2012. Do institutional changes affect business cycles? Evidence from Europe. J. Econ. Dynam. Contr. 36 (10), 1520-1533.

Clark, T.E., van Wincoop, E., 2001. Borders and business cycles. J. Int. Econ. 55, 59-85.

Ciccarelli, M., Ortega, E., Valderrama, M., 2016. Commonalities and cross-country spillovers in macroeconomic-financial linkages. B. E. J. Macroecon. 16 (1), 231-275.

Croux, C., Forni, M., Reichlin, L., 2001. A measure of comovement for economic variables: theory and empirics. Rev. Econ. Stat. 83 (2), 232-241.

de Haan, J., Inklaar, R., Richard, J., 2008. Will business cycles in the Euro Area converge: a critical survey of empirical research. J. Econ. Surv. 22 (2), 234-273.

Del Negro, M., Otrok, C., 2008. Dynamic Factor Models with Time-varying Parameters: Measuring Changes in International Business Cycles. Federal Reserve Bank of New York Staff Reports no. 326.

Dickey, D., Fuller, W., 1979. Distribution of the estimators for autoregressive time series with a unit root. J. Am. Stat. Assoc. 74, 427-431.

Ductor, D., Leiva-Leon, D., 2016. Dynamics of global business cycle interdependence. J. Int. Econ. 102, 110-127.
Dufrenot, G., Keddad, B., 2014. Business cycles synchronization in East Asia: a Markov-switching approach. Econ. Modell. 42, 186-197.

Fatas, A., 1997. Countries or regions? Lessons from the EMS experience. Eur. Econ. Rev. 41, 743-751.

Gadea, M.D., Gomez-Loscos, A., Montañes, A., 2012. Cycles inside cycles: Spanish regional aggregation. SERIEs, Spanish Econ. Assoc. 3 (4), 423-456.

Gadea-Rivas, M., Gómez-Loscos, A., Bandres, E., 2018. Clustering regional business cycles. Econ. Lett. 162 (C), 171-176.

Giannone, D., Lenza, M., Reichlin, L., 2010. In: Alesina, A., Giavazzi, F. (Eds.), Business Cycles in the Euro Area in Europe and the Euro. The University of Chicago Press, pp. $141-167$.

Guerin, P., Leiva-Leon, D., 2017. Model averaging in Markov-switching models: predicting national recessions with regional data. Econ. Lett. 157, 45-49, Elsevier.

Hamilton, J., Owyang, M., 2012. The propagation of regional recessions. Rev. Econ. Stat. 94 (4), 935-947.

Harding, D., Pagan, A., 2006. Synchronization of cycles. J. Econom. 132 (1), 59-79.

Herrendorf, B., Rogerson, R., Valentinyi, A., 2014. (Chapter 6). Growth and Structural Transformation. Handbook of Economic Growth, vol. 2, pp. 855-941.

Imbs, J., 2004. Trade, finance, specialisation and synchronisation. Rev. Econ. Stat. 86 (3), 723-734.

Kim, C., Nelson, C.R., 1999. State-Space Models with Regime Switching: Clasical and Gibbs-Sampling Approaches with Applications. MIT press.

Koop, G., 2014. Forecasting with dimension switching VARs. Int. J. Forecast. 30 (2), 280-290.

Koop, G., Korobilis, D., 2012. Forecasting inflation using dynamic model averaging. Int. Econ. Rev. 53 (3), 867-886.

Koop, G., Korobilis, D., 2013. Large time-varying parameter VARs. J. Econom. 177 (2), 185-198.

Kose, M.A., Otrok, C., Prasad, E., 2012. Global business cycles: convergence or decoupling? Int. Econ. Rev. 53 (2), 511-538.

Lee, J., 2012. Measuring business cycle comovements in europe: evidence from a dynamic factor model with time-varying parameters. Econ. Lett. 115 (3), 438-440 2012.

Lee, J., 2013. Business cycle synchronization in europe: evidence from a dynamic factor model. Int. Econ. J. 7 (3), 347-364.

Leiva-Leon, D., 2016. Measuring Business Cycles Intra-Synchronization in US. A Regime-Switching Interdependence Framework. Oxf. Bull. Econ. Stat. 79 (4), 513-545.

Liu, Ch A., 2011. Averaging Estimators for Kernel Regressions. University of Wisconsin-Madison Working Paper. 
Marino, F., 2013. Regional Fluctuations and National Cohesion in the EU12: a Pre-Maastricht Assessment, SERIES Working Papers, vol. 48. University of Bari.

Ng, S., Perron, P., 2001. Lag Length Selection and the Construction of Unit Root Tests with Good Size and Power. Econometrica 69 (6), 1519-1554.

Ozyurt, S., Dees, S., 2015. Regional Dynamics of Economic Performance in the EU: to what Extent Spatial Spillovers Matter? European Central Bank Working Paper 1870.

Phillips, K., 1991. A Two-Country Model of Stochastic Output with Changes in Regime. J. Int. Econ. 3, 121-142.

Phillips, P.C.B., Moon, H.R., 1999. Linear Regression Limit Theory for Nonstationary Panel Data. Econometrica 67 (5), 1057-1111.
Raftery, A., Karny, M., Ettler, P., 2010. Online Predictions Under Model Uncertainty Via Dynamic Model Averaging: Application to a Cold Rolling Mill. Technometrics 52, 52-66.

Ramajo, J., Marquez, M.A., Hewings, G., Salinas, M.M., 2008. Spatial heterogeneity and interregional spillovers in the European Union: Do cohesion policies encourage convergence across regions? Eur. Econ. Rev. 52, 551-567.

Rodriguez-Pose, A., 2013. Do Institutions Matter for Regional Development? Reg. Stud. 47 (7), 1034-1047.

Sala-i-Martin, X., 1996. Regional cohesion: Evidence and theories of regional growth and convergence. Eur. Econ. Rev. 40, 1325-1352.

Stone, C.J., 1977. Consistent nonparametric regression. Ann. Stat. 5, 595-620. 